\title{
EILENBERG-MOORE MODELS FOR FIBRATIONS
}

BY

\author{
J. C. THOMAS
}

\begin{abstract}
E. M. model is a new invariant in rational homotopy theory which gives us both a Künneth object and a Tate-Josefiak resolution. With the E. M. model, we study relations between formality of base, total space and fibre of a Serre fibration, obstructions to k-realizability of a cohomology equivalence between two continuous maps and formalizable maps.
\end{abstract}

I. Introduction. The main notion of this paper is that of E. M. model of a fibration. This is a new invariant of the rational homotopy theory of Serre fibrations, with a filtered structure which gives us both a Künneth object in the sense of Gugenheim and May and a Tate-Josefiak resolution of the cohomology of the total space of the fibration. The filtration itself is a homotopy invariant and induces the EilenbergMoore spectral sequence of the fibration. Since we work in commutative differential graded algebras over a field $\mathbf{k}$ of characteristic zero, this E. M. model is easily computable with the aid of the Sullivan theory of P. L. forms and minimal models. We adopt the terminology of [6] (see also [7, 14]). Existence and uniqueness of the E. M. model is stated in

ThEOREM. Suppose that $\gamma:\left(B, d_{B}\right) \rightarrow\left(C, d_{C}\right)$ is a homomorphism of c.d.g.a.'s, and $H^{0}\left(B, d_{B}\right)=H^{0}\left(C, d_{C}\right)=\mathbf{k}$.

(A) There exists a K. S. model $(\mathcal{F}, \eta)[6]$

$$
\begin{aligned}
& \left(B, d_{B}\right) \stackrel{\gamma}{\rightarrow} \quad\left(C, d_{C}\right) \\
& \| \quad \uparrow \eta \\
& \mathscr{F}:\left(B, d_{B}\right) \quad \stackrel{\iota}{\rightarrow}(B \otimes \Lambda Y, D) \stackrel{\rho}{\rightarrow} \quad(\Lambda Y, \bar{D})
\end{aligned}
$$

in which:

(1) $Y$ has a second gradation $Y=\bigotimes_{k \geqslant 0} Y_{k}$.

(2) $D_{\mid Y}$ lowers the second gradation (so $D=d_{B} \oplus 1+D_{1}+D_{2}+\cdots$ where $\left.D_{i}\left(Y_{p}\right) \subset B \otimes(\Lambda Y)_{p-i}\right)$.

(3) The induced $\bar{D}_{1}=\rho D_{1}$ is decomposable.

(4) In the spectral sequence determined by the second gradation $\left(E_{1}^{-p, q}=(H(B) \otimes\right.$ $\left.\left.(\Lambda Y)_{p}\right)^{q-p}\right)$ we have $E_{2}^{-p, q}=0$ for $p \neq 0$ and therefore $\eta^{*}: E_{2}^{0, q} \rightarrow H^{q}\left(C, d_{C}\right)$ is an isomorphism.

Received by the editors March 25, 1981 and, in revised form, September 17, 1981.

1980 Mathematics Subject Classification. Primary 55P62, 55T20.

Key words and phrases. Eilenberg-Moore spectral sequence, Serre fibration, rational homotopy, K. S. models, formal maps.

'ERA au CNRS 07590. 
(B) Suppose there exists a second $K . S$. model $\left(\mathscr{F}^{\prime}, \eta^{\prime}\right)$ which verifies (1), (2),(3), (4). Then there exists an isomorphism of $K S$ extensions (Id, $\varphi, \bar{\varphi})$ between $\mathcal{F}$ and $\mathcal{F}^{\prime}$ such that:

(i) $\varphi\left(B \oplus(\Lambda Y)_{<p}\right)=B \otimes\left(\Lambda Y^{\prime}\right)_{<p}$.

(ii) $\eta^{\prime}$ is homotopic to $\eta \circ \varphi($ rel $B)$.

Since $(B \otimes \Lambda Y, D)$ is a distinguished split object in the sense of Gugenheim-May, $H(\Lambda Y, \bar{D})$ is naturally isomorphic to $D$ tor $_{B}(\mathbf{k}, C)$ and we have the geometric interpretation:

THEOREM. Consider a Serre fibration of path connected spaces

$$
F \stackrel{j}{\rightarrow} E \stackrel{\pi}{\rightarrow} M
$$

in which one of $H^{*}(M), H^{*}(F)$ is a graded space of finite type and $\pi_{1}(B)$ acts nilpotently in each $H^{p}(F)$. Let $(\mathscr{F}, \eta)$ be the $E$. M. model of $A(\pi): A(M) \rightarrow A(E)$ where $A$ is the Sullivan functor.

(i) $(\Lambda Y, \bar{D})$ is a K.S. model of $F$ (in general neither minimal nor filtered model in the sense of Halperin-Stasheff [8]).

(ii) For any K. S. model $\gamma: B \rightarrow C$ of $\pi$ with $B$ and $C$ connected c.d.g.a.'s, $H^{*}(F)$ is isomorphic to $D$ tor $_{B}(\mathbf{k}, C)$.

The advantage of the E. M. model is that it is easily computable since $D$ is a perturbation of the underlying formal model $(\mathcal{G}, \xi)$ of $(\mathscr{F}, \eta)$.

$(\mathcal{G}, \xi)$ is a K. S. minimal model of $\gamma^{*}[6$ or 7$]$

$$
\begin{aligned}
& H^{*}(B) \stackrel{\gamma^{*}}{\rightarrow} \quad H^{*}(C) \\
& \| \quad \uparrow \xi \\
& H^{*}(B) \stackrel{\iota_{1}}{\rightarrow}\left(H(B) \otimes \Lambda Y, D_{1}^{*}\right) \stackrel{\rho_{1}}{\rightarrow}\left(\Lambda Y, \bar{D}_{1}^{*}\right)
\end{aligned}
$$

with $\left(H(B) \otimes \Lambda Y, D_{1}^{*}\right)$ a Tate-Josefiak resolution of $H^{*}(C)$ as $H(B)$-algebra.

From definitions and uniqueness, we can see that the E. M. model of the natural inclusion $(\mathbf{k}, 0) \hookrightarrow\left(C, d_{C}\right)$ is the filtered model of Halperin-Stasheff and that the underlying formal model is just the bigraded model in the sense of [8].

The spectral sequence determined by the second gradation of an E. M. model will be called the algebraic Eilenberg-Moore spectral sequence. Now given a pull back of a rational fibration (with simply connected bases) there is associated the classical spectral sequence of Eilenberg-Moore [2]. In [15] Vigue shows how to obtain this from Sullivan's model theory. Her construction, however, yields an E. M. model, and thus the two spectral sequences coincide.

The Vigue filtration is finer than our filtration and requires the H. S. filtration in a filtered model of the base. We give a direct proof of the following result of [15].

Proposition. Let (*) be as in the preceding theorem. If $M$ is 1-connected the following conditions are equivalent:

(i) $(\Lambda Y, \bar{D})$ is a filtered model of $F$ (in the sense of [8]). 
(ii) $\left(\Lambda Y, \bar{D}_{1}\right)$ is a bigraded model of $F$ (in the sense of [8]).

(iii) The Serre spectral sequence collapses at the $E_{2}$ level $\left(d_{r}=0, r \geqslant 2\right)$.

Recall that a space is formal [8] if its filtered model is isomorphic to its bigraded model. From the preceding result, if $j^{*}$ is surjective then the geometric realization of $\left(\Lambda Y, \bar{D}_{1}\right)$ (the formal fibre) is a formal space. The converse is false.

Proposition. Let $(*) \stackrel{j}{\rightarrow} E \stackrel{\pi}{\rightarrow} M$ as in the preceding theorem.

(i) If $M$ and $F$ are formal spaces and if $\pi_{\psi}(E)=\pi_{\psi}(F) \oplus \pi_{\psi}(M)$ then the $E$. $M$. spectral sequence $[8]$ of the space $E$ collapses at the $E_{2}$ level.

(ii) If $E$ is a formal space and if $H^{*}(E) \cong H(M) \otimes H(F)$ as k-algebras and $H(M)$-modules then the $E$. $M$. spectral sequences of the spaces [8] $M$ and $F$ collapse at the $E_{2}$ level.

(iii) If $E, F, M$ are nilpotent spaces, $E$ and $F$ formal spaces and $H(E) \cong H(M) \otimes$ $H(F)$ as k-algebras and $H(M)$-modules, then the rationalized fibration

$$
F_{\mathbf{Q}} \rightarrow E_{\mathbf{Q}} \rightarrow M_{\mathbf{Q}}
$$

is trivial.

We exhibit a fibration having the following properties.

$F$ and $M$ are both formal and coformal [9].

$\pi_{\psi}(E)=\pi_{\psi}(M) \oplus \pi_{\psi}(F)$ and $H(E) \cong H(M) \otimes H(F)$ as algebras and $H(M)$ modules.

The rationalized fibration is not trivial and the total space is not formal.

Then we use E. M. models to construct obstructions to realize a cohomology equivalence between two maps by a rational homotopy equivalence. These obstructions are independent of the field $\mathbf{k}$ and permit us to study formalizable maps. In particular we obtain the following sequence of strict implications for (*),

( $\pi$ is formalizable $) \Rightarrow(F$ has the same rational homotopy type as the formal fibre) $\Rightarrow$ (The E. M. spectral sequence of $(*)$ collapses at the $E_{2}$ level).

We prove that these obstructions depend effectively on the homotopy class of the formalization of the base, and treat some examples.

The paper is organized as follows.

§II. Algebraic constructions. In which we prove existence and uniqueness of the E. M. model and of the underlying formal model of a c.d.g.a. homomorphism.

§III. Geometric interpretation and examples. In which we first make precise the relation between fibrations and E. M. models $(1,2,3)$, and give some examples $(4,5,6)$. Then we study E. M. models of T.N.C.Z., H. T., C. T. fibrations $(7,8)$. In $(9,10)$ we look at the E. M. spectral sequence and finally in (11) we study relations between formality of base, total space and fibre.

$\S I V$. Obstruction to k-realizibility. With the E. M. model, we give a relative version of [8] and treat some examples.

$\S \mathrm{V}$. Formalizable maps. First we apply obstruction to k-realizibility, in order to study formalizable map. In the particular case when $H\left(A, d_{A}\right)$ is a free c.g.a., we develop a new class of computable obstructions which vanish if and only if the 
homomorphism $\left(A, d_{A}\right) \rightarrow\left(C, d_{C}\right)$ is formal. Finally, we look at the E. M. fibre and the formal fibre of a formalizable map.

The author is deeply indebted to S. Halperin and J. D. Stasheff for their helpful advice and for suggestions for improving the presentation of this paper.

\section{Algebraic constructions.}

II.1. Let $\left(B, d_{B}\right)$ and $\left(C, d_{c}\right)$ be two augmented c.d.g.a.'s (commutative differential graded algebras over $\mathbf{k})$ and suppose $H^{0}\left(B, d_{B}\right)=H^{0}\left(C, d_{C}\right)=\mathbf{k}$ and let $\gamma$ : $\left(B, d_{B}\right) \rightarrow\left(C, d_{C}\right)$ be a homomorphism of augmented c.d.g.a.'s. Let $m:\left(B^{\prime}, d_{B^{\prime}}\right) \rightarrow$ $\left(B, d_{B}\right)$ be any model of $B$. ( $B^{\prime}$ can always be chosen 0 -connected.)

II.2. An E. M. model of $\gamma$ with base $\left(\left(B^{\prime}, d_{B^{\prime}}\right), m\right)$ is a K. S. model $(\mathcal{F}, \eta)[6]$ of $\gamma$,

$$
\begin{aligned}
& \left(B, d_{B}\right) \quad \stackrel{\gamma}{\rightarrow} \quad\left(C, d_{c}\right) \\
& m \uparrow \quad \uparrow \eta \\
& \mathscr{F}:\left(B^{\prime}, d_{B^{\prime}}\right) \quad \underset{\imath}{\rightarrow}\left(B^{\prime} \otimes \Lambda Y, D\right) \underset{\rho}{\vec{\rho}}(\Lambda Y, \bar{D})
\end{aligned}
$$

in which:

(1) $Y$ has a second gradation $Y=\otimes_{k \geqslant 0} Y_{k^{\prime}}$.

(2) $D_{\mid Y}$ lowers the second gradation (so $D=d_{B} \otimes 1+D_{1}+D_{2}+\cdots$ where $\left.D_{i}\left(Y_{p}\right) \subset B^{\prime} \otimes(\Lambda Y)_{p-i}\right)$.

(3) The induced $\bar{D}_{1}=\rho D_{1}$ is decomposable.

(4) In the spectral sequence determined by the second gradation (i.e. $E_{1}^{-p, q}=$ $\left.\left(H(B) \otimes(\Lambda Y)_{p}\right)^{q-p}, p, q \geqslant 0\right)$ we have $E^{-p, q}=0$ for $p \neq 0$.

II.3. From the preceding definition, we have $H\left(B^{\prime} \otimes \Lambda Y, D\right)=E_{2}^{0, *}$,

$$
\eta^{*}: E_{2}^{0, q} \rightarrow H^{q}\left(C, d_{C}\right)
$$

is an isomorphism for each $q \geqslant 0$. Let $\bar{D}$ denote the induced differential on $\Lambda Y$ :

$$
\bar{D}=\bar{D}_{1}+\bar{D}_{2}+\bar{D}_{3}+\cdots
$$

with $\bar{D}_{i}\left(Y_{p}\right) \subset(\Lambda Y)_{p-i}$.

II.4. Consider on $\left(B, d_{B}\right)$ (resp. $\left.\left(B^{\prime}, d_{B}\right),\left(C, d_{C}\right)\right)$ the trivial decreasing filtration and on $B^{\prime} \otimes \Lambda Y$ (resp. on $\Lambda Y$ ) the filtration $F_{p}=F^{-p}=B \otimes(\Lambda Y)_{\leqslant p}$ (resp. $\left.\bar{F}_{p}=\bar{F}^{-p}=(\Lambda Y)_{\leqslant p}\right)$. Thus we have at the $E_{1}$ level of the corresponding spectral sequences, for $p=0$,

$$
\begin{array}{ccc}
H\left(B, d_{B}\right) & \stackrel{\gamma^{*}=\gamma_{1}^{0, *}}{\rightarrow} & H\left(C, d_{C}\right) \\
m^{*}=m_{1}^{0 . *} \uparrow \cong & & \uparrow \eta_{1}^{0, *} \\
H\left(B^{\prime}, d_{B^{\prime}}\right) & \stackrel{\iota_{1}^{0, *}}{\rightarrow} & H\left(B^{\prime}, d_{B^{\prime}}\right) \otimes \Lambda Y_{0} \stackrel{\rho_{1}^{0, *}}{\rightarrow} \Lambda Y_{0}
\end{array}
$$

and for $p>0$,

$$
\begin{aligned}
& \begin{array}{ll}
0= & 0 \\
\| & \uparrow \eta_{1}^{-p, *}
\end{array} \\
& 0 \quad \rightarrow \quad H\left(B^{\prime}, d_{B^{\prime}}\right) \otimes(\Lambda Y)_{p} \stackrel{\rho_{1}^{-p, *}}{\rightarrow} \quad(\Lambda Y)_{p}
\end{aligned}
$$


In this way we obtain the commutative diagram

$$
\begin{aligned}
& H\left(B, d_{B}\right) \quad \stackrel{\gamma^{*}}{\rightarrow} \quad H\left(C, d_{C}\right) \\
& m^{*} \uparrow \\
& \mathcal{G}: H\left(B^{\prime}, d_{B^{\prime}}\right) \stackrel{\stackrel{\iota}{\rightarrow}}{\rightarrow}\left(H\left(B^{\prime}, d_{B^{\prime}}\right) \otimes \Lambda Y, D_{1}^{*}\right) \stackrel{\rho_{1}}{\rightarrow} \quad\left(\Lambda Y, \bar{D}_{1}\right)
\end{aligned}
$$

where $\zeta_{\mid \Lambda Y_{0}}=\eta_{1}^{0, *}{ }_{\mid \Lambda Y_{0}}$ and $\zeta_{\mid(\Lambda Y)_{>1}}=0 . \zeta^{*}$ is an isomorphism and since $\bar{D}_{1}$ is decomposable the pair $(\mathcal{G}, \zeta)$ is a K. S. minimal model of $\gamma^{*}$ with base $H\left(B^{\prime}, d_{B}\right)$ [6].

II.5. The pair $(\mathcal{G}, \zeta)$ will be called the underlying formal model of $(\mathcal{F}, \eta)$. The complex $\left(E_{1}, D_{1}^{*}\right)$ is a free resolution of $H\left(C, d_{C}\right)$ so that we see directly from the definition that $\left(B^{\prime} \otimes \Lambda Y, D\right)$ is Künneth object [5]. Therefore $D$ tor $_{B}(\mathbf{k}, C)$ is naturally isomorphic to $H\left(\mathrm{k} \otimes B^{\prime} \otimes_{B^{\prime}} Y, 1 \otimes_{B^{\prime}} D\right)$ which is isomorphic to $H(\Lambda Y, \bar{D})$. If we denote $\bar{E}_{r}$ the spectral sequence induced on $(\Lambda Y, \bar{D})$ by $\bar{F}_{p}$, with the notations of [5]

$$
E_{r}(\mathbf{k}, B, C) \simeq \bar{E}_{r}
$$

is the algebraic Eilenberg-Moore spectral sequence.

This justifies the name E. M. model.

II.6. Theorem. Suppose that $H^{0}\left(B, d_{B}\right)=H^{0}\left(C, d_{C}\right)=\mathbf{k}$ and $\gamma:\left(B, d_{B}\right) \rightarrow$ $\left(C, d_{C}\right)$ is a c.d.g.a. homomorphism. Then $\gamma$ admits an E. M. model.

We prove this result in the following sections $(7,8,9,10)$.

II.7. Construction of $(\mathscr{F}, \eta)$. We proceed by induction on the second gradation. Let $\left(B^{\prime}, d_{B}\right) \stackrel{m}{\rightarrow}\left(B, d_{B}\right)$ be a model of $\left(B, d_{B}\right)$

(a) $k=0$ : Consider a linear section $s$ of the composite

$$
\begin{aligned}
C^{+} \cap \operatorname{ker} d_{c} & \rightarrow H^{+}\left(C, d_{c}\right) \\
& \rightarrow H^{+}\left(C, d_{c}\right) /\left(H^{+}\left(C, d_{c}\right) \cdot H^{+}\left(C, d_{c}\right)+\gamma^{*}\left(H^{+}\left(B, d_{B}\right)\right)\right)
\end{aligned}
$$

and put

$$
\begin{gathered}
Y_{0}=H^{+}\left(C, d_{C}\right) /\left(H^{+}\left(C, d_{C}\right) \cdot H^{+}\left(C, d_{C}\right)+\gamma^{*}\left(H^{+}\left(B, d_{B}\right)\right)\right), \\
D=d_{B^{\prime}} \otimes 1 \quad \text { in } B^{\prime} \otimes \Lambda Y_{0}, \\
\eta_{0}(b \otimes 1)=\gamma m(b), \quad \eta_{0}(1 \otimes y)=s(y),
\end{gathered}
$$

so that

$$
\eta_{0}^{*}: H\left(B^{\prime} \otimes \Lambda Y_{0}, D\right) \rightarrow H\left(C, d_{C}\right)
$$

is surjective. (For the sake of simplicity we will denote $H_{0}$ for $H\left(B \otimes \Lambda Y_{0}, D_{0}\right)$.)

(b) $k=1$ : Set $K_{0}=\operatorname{ker} \eta_{0}^{*}$ and let $\pi_{0}: B^{\prime} \otimes \Lambda Y_{0} \cap \operatorname{ker} D_{0} \rightarrow H_{0}, p_{0}: K_{0} \rightarrow$ $K_{0} / K_{0} H_{0}^{+}$be the canonical projections. Write $L_{0}=\pi_{0}^{-1}\left(K_{0}\right)$, let $s_{1}$ be a linear section of $p_{0} \circ \pi_{0 \mid L_{0}}$ and put $\sigma_{1}=\pi_{0} \circ s_{1}$. Remark that $K_{0}^{0}=0$ and $K_{0}=\operatorname{Im} \sigma_{1} \oplus$ $K_{0} H_{0}^{+}$, whence $K_{0}=H_{0} \cdot \operatorname{Im} \sigma_{1}$. We put $Y_{1}=K_{0} / K_{0} \cdot H_{0}^{+}$with the convention $y \in Y_{1}$ equals $\bar{y} \in K_{0} / K_{0} H_{0}^{+}$but $|y|=|\bar{y}|-1$, and extend $D$ to $B^{\prime} \otimes \Lambda Y_{0} \otimes \Lambda Y_{1}$ by putting

$$
D(1 \otimes y)=s_{1}(\bar{y}) \in B^{\prime} \otimes \Lambda Y_{0}
$$


One verifies that $\eta_{0}(D(1 \otimes y))=d_{C} a$, so that we can extend $\eta_{0}$ to $\eta_{1}$

$$
\eta_{1}:\left(B^{\prime} \otimes \Lambda Y_{0} \otimes \Lambda Y_{1}, D\right) \rightarrow\left(C, d_{C}\right) .
$$

It follows as in [7, Lemma 6.9] that $B^{\prime} \otimes \Lambda Y_{0} \otimes \Lambda Y_{1}$ is $c$-connected.

(c) Suppose we have defined $Y_{<k}, D$ and $\eta_{k}$ such that

(·) $\eta_{k \mid B^{\prime} \otimes \Lambda Y_{<k-1}}=\eta_{k-1}$,

(・) $D Y_{i} \subset B \otimes\left(\Lambda Y_{<k}\right)_{<i}$,

$(\cdots)\left(B^{\prime} \otimes \Lambda Y_{<k}, D\right)$ is $c$-connected and consider the exact sequence

$$
0 \rightarrow K_{k} \rightarrow F_{k}\left(H\left(B^{\prime} \otimes \Lambda Y_{<k}\right)\right) \stackrel{\eta_{k}^{*}}{\rightarrow} H\left(C, d_{C}\right) \rightarrow 0
$$

where $F_{p}\left(H\left(B^{\prime} \otimes \Lambda Y_{<k}\right)\right)$ is the obvious filtration. Let $\pi_{k}: F_{k}\left(B^{\prime} \otimes \Lambda Y_{\leqslant k}\right) \cap \operatorname{ker} D$ $\rightarrow F_{k} H\left(B^{\prime} \otimes \Lambda Y_{<k}, D\right)$ and $p_{k}: K_{k} \rightarrow K_{k} / K_{k} \cdot H_{0}^{+}$be the canonical projections, put $L_{k}=\pi_{k}^{-1}\left(K_{k}\right)$, let $s_{k+1}$ be a linear section of $p_{k} \circ \pi_{k \mid L_{k}}$ and set $\sigma_{k+1}=\pi_{k} \circ s_{k+1}$. Put $Y_{k+1}=K_{k} / K_{k} \cdot H_{0}^{+}$with the convention $y \in Y_{k+1}$ equals $\bar{y} \in K_{k} / K_{k} \cdot H_{0}^{+}$but $|y|=|\bar{y}|-1$, and extend $D$ to $B^{\prime} \otimes \Lambda Y_{<k+1}$ by

$$
D(1 \otimes y)=s_{k+1}(\bar{y}) \in F_{k}\left(B \otimes \Lambda Y_{<k}\right) \cap \operatorname{ker} D .
$$

Extend $\eta_{k}$ to $\eta_{k+1}$ as above. Again Lemma 6.9 of [7] shows that $B^{\prime} \otimes \Lambda Y_{\leqslant k+1}$ is $c$-connected.

(d) By this process, we define a K. S. extension $\mathscr{F}$ which is a model of $\gamma$ (since $\eta^{*}$ is an isomorphism) such that $D Y_{i} \subset B^{\prime} \otimes F_{i}(\Lambda Y)$. The two following lemmas prove that $(\mathscr{F}, \eta)$ is an E. M. model.

II.8. LEMMA. In the preceding construction, for the spectral sequence induced on $(B \otimes \Lambda Y, D)$ by the second gradation, we have $E_{2}^{-p, *}=0$, if $p>0$.

II.9. Proof of Lemma II.8. By a standard spectral sequence argument Lemma II.8 is equivalent to $\left\langle\right.$ For any $k \geqslant 1$ and any $\Phi \in B \otimes(\Lambda Y)_{<k} \cap \operatorname{ker} D$, there exists $w \in B \otimes(\Lambda Y)_{<k+1}$ such that $\left.\left.\Phi-D w \in B \otimes \Lambda Y_{0}\right\rangle\right\rangle$.

Let $\Phi \in B \otimes(\Lambda Y)_{<k} \cap \operatorname{ker} D$ represent $[\Phi] \in F_{k} H\left(B \otimes \Lambda\left(Y_{<k}\right)\right)$. By construction, there exists $\alpha \in B \otimes \Lambda Y_{0}$ such that $\eta_{0}^{*}([\alpha])=\eta_{k}^{*}([\Phi])$. Therefore,

$$
[\Phi-\alpha] \in K_{k}=\operatorname{Im} \sigma_{k+1} \oplus H_{0}^{+} K_{k}=H_{0} \cdot \operatorname{Im} \sigma_{k+1},
$$

so that we can write $[\Phi-\alpha]=\sum_{i} \lambda_{i} \sigma\left(\gamma_{i}\right)$ with $\lambda_{i} \in H_{0}$ and $\gamma_{i} \in Y_{k+1}$. From the definition of $D, \Phi-\alpha=D\left(\Sigma_{i} \omega_{i} \lambda_{i}\right)+D u$ with $\omega_{i} \in B \otimes \Lambda Y_{0}$ and $u \in F_{k}$. Put $w=\sum \omega_{i} \gamma_{i}+u$.

II.10. LEMMA. In the preceding construction, $\bar{D}_{1}$ is decomposable.

II.11. Proof of II.10. Suppose there exists $y \in Y_{k+1}$ such that $\bar{D}_{1} y$ is not decomposable. The decomposition of $D y$ given by

$$
\begin{aligned}
D y \in F_{k}= & \left(\mathbf{k} \otimes Y_{k}\right) \oplus\left(\left(B \otimes \Lambda Y_{0}\right)^{+} \otimes Y_{k}\right) \\
& \oplus\left(\bigoplus_{\substack{i+j=k \\
i, j>0}}\left(B \otimes(\Lambda Y)_{i}\right) \cdot\left(B \otimes(\Lambda Y)_{j}\right)\right)
\end{aligned}
$$


will be written $D y=x-\sum_{i} \omega_{0}^{i} z_{i}-\Omega$. From the relation $D \circ D=0$, we have

$$
D x=\sum_{i} D_{0} \omega_{0}^{i} \cdot z_{i}+\sum_{i}(-1)^{\left|\omega_{0}^{i}\right|} \omega_{0}^{i} D z_{i}+D \Omega \text {. }
$$

Since $x$ and $z_{i}$ belong to $Y_{k}$ and $D x \in B^{\prime} \otimes(\Lambda Y)_{k-1}$ necessarily $D_{0} \omega_{0}^{i}=0$. Since $D \Omega \in B \otimes(\Lambda Y)_{<k-1}$, projecting on $F_{k-1}\left(H\left(B^{\prime} \otimes \Lambda Y_{<k-1}, D\right)\right)$ yields the relation

$$
[D x]=\left[\sum_{i}(-1)^{\left|\omega_{0}^{i}\right|} \omega_{0}^{i} D z_{i}\right]=\sum_{i}(-1)^{\left|\omega_{0}^{i}\right|}\left[\omega_{0}^{i}\right]\left[D z_{i}\right]
$$

whence

$$
\sigma_{k}(x)=\sum_{i}(-1)^{\left|\omega_{0}^{i}\right|}\left[\omega_{0}^{i}\right] \sigma_{k}\left(z_{i}\right) \in \operatorname{Im} \sigma_{k} \cap H_{0}^{+} \operatorname{Im} \sigma_{k} \subset \operatorname{Im} \sigma_{k} \cap H_{0}^{+} K_{k}=0 .
$$

This implies $x=0$.

Since the underlying formal model of an E. M. model for $\gamma: B \rightarrow C$ is the minimal model for $\gamma^{*}: H(B) \rightarrow H(C)$ we obtain from $[7,6.16]$

II.12. Proposition. If $\gamma^{*}: H\left(B, d_{B}\right) \rightarrow H^{*}\left(C, d_{C}\right)$ is n-regular (i.e. $\gamma^{*}$ isomorphism until degree $n$ and injective in degree $n+1)$ then $\Lambda Y$ is n-connected.

Exactly as in $[8, \S 3]$, we have

II.13. Proposition. Any two of the following three conditions implies the third:

(i) $H\left(C, d_{C}\right)$ is a noetherian algebra (resp. graded space of finite type).

(ii) $H\left(B, d_{B}\right)$ is a noetherian algebra (resp. graded space of finite type).

(iii) For each $k, Y_{k}$ is finite dimensional (resp. $Y$ is a graded space of finite type).

First, we prove uniqueness of underlying formal model.

II.14. THEOREM. If $\left(\mathcal{F}^{\prime}, \eta^{\prime}\right)$ and $\left(\mathscr{F}^{\prime \prime}, \eta^{\prime \prime}\right)$ are two $E$. M. models of $\gamma$, with same base $\left(\left(B^{\prime}, d_{B^{\prime}}\right), m\right)$, and underlying formal models $\left(\mathcal{G}^{\prime}, \zeta^{\prime}\right)$ and $\left(\mathcal{G}^{\prime \prime}, \zeta^{\prime \prime}\right)$ then there exists a $K$. $S$. extension isomorphism ( $\left.\operatorname{Id}_{H\left(B^{\prime}, d_{B^{\prime}}\right)}, \varphi, \bar{\varphi}\right)$ between $\mathcal{G}^{\prime}$ and $\mathcal{G}^{\prime \prime}$ such that:

(a) $\varphi$ and $\bar{\varphi}$ are of bidegree $(0,0)$,

(b) $\zeta^{\prime \prime} \circ \varphi=\zeta^{\prime}$.

II.15. Proof OF II.14. Fix notation by the following diagram

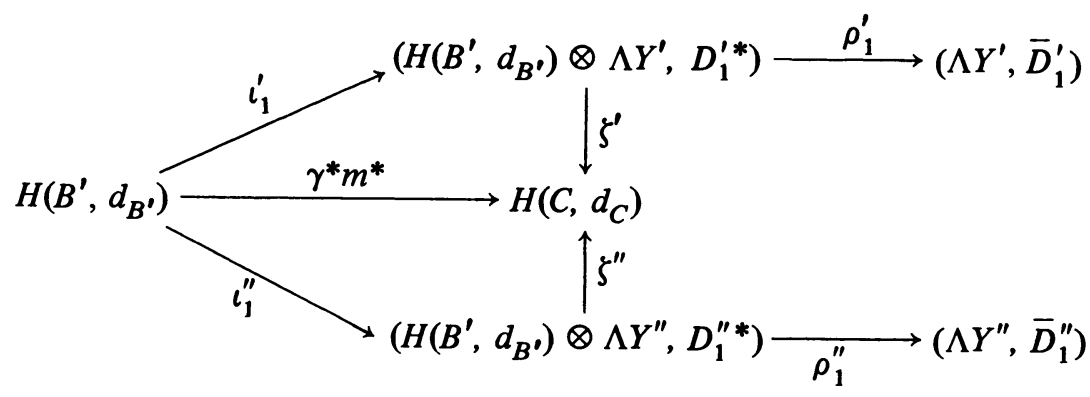

We construct $\varphi$ by induction on second gradation:

(a) $k=0$ : Put $\varphi(\beta \otimes 1)=\beta \otimes 1, \beta \in H\left(B^{\prime}, d_{B^{\prime}}\right)$. If $y_{0} \in Y_{0}^{\prime}$, there exists $\alpha_{y_{0}} \in$ $H\left(B^{\prime}\right) \otimes \Lambda Y_{0}^{\prime \prime}$ such that $\zeta^{\prime}\left(y_{0}\right)=\zeta^{\prime \prime}\left(\alpha_{y_{0}}\right)$. Thus we may extend $\varphi$ to a c.d.g.a. 
homomorphism of bidegree $(0,0) \varphi:\left(H\left(B^{\prime}\right) \otimes \Lambda Y_{0}^{\prime}, 0\right) \rightarrow\left(H\left(B^{\prime}\right) \otimes \Lambda Y_{0}^{\prime \prime}, 0\right)$ such that $\zeta^{\prime \prime} \circ \varphi=\zeta^{\prime}$.

(b) $k=1$ : Let $y_{1} \in Y_{1}$, then $\varphi\left(D_{1}^{\prime *} y_{1}\right) \in H\left(B^{\prime}\right) \otimes \Lambda Y_{0}^{\prime \prime}$ and $\zeta^{\prime \prime} \circ \varphi\left(D_{1}^{\prime *} y_{1}\right)=$ $\zeta^{\prime}\left(D_{1}^{\prime *} y_{1}\right)=0$. Therefore, there exists $\alpha_{y_{1}} \in H(B) \otimes\left(\Lambda Y^{\prime \prime}\right)_{1}$ such that $\varphi\left(D_{1}^{\prime *} y_{1}\right)=$ $\left(D_{1}^{\prime \prime *} \alpha_{y_{1}}\right)$. As usual we may extend $\varphi$ to a c.d.g.a. homomorphism of bidegree $(0,0)$

$$
\varphi:\left(H\left(B^{\prime}\right) \otimes \Lambda Y_{<1}^{\prime}, D_{1}^{\prime *}\right) \rightarrow\left(H\left(B^{\prime}\right) \otimes \Lambda Y_{\leqslant 1}^{\prime \prime}, D_{1}^{\prime \prime *}\right)
$$

such that $\zeta^{\prime \prime} \circ \varphi=\zeta^{\prime}$.

(c) $k \geqslant 2$ : Let $y_{k} \in Y_{k}$, then $\varphi\left(D_{1}^{\prime *} y_{k}\right) \in H\left(B^{\prime}\right) \otimes\left(\Lambda Y^{\prime \prime}\right)_{k-1} \cap \operatorname{ker} D_{1}^{\prime \prime *}$. Since $E_{2}^{k-1,0}=0$, there exists $\alpha_{y_{k}} \in H\left(B^{\prime}\right) \otimes\left(\Lambda Y^{\prime \prime}\right)_{k}$ such that $\varphi\left(D_{1}^{\prime *} y_{k}\right)=D_{1}^{\prime \prime *} \alpha_{y_{k}}$.

(d) Finally, we obtain a commutative diagram

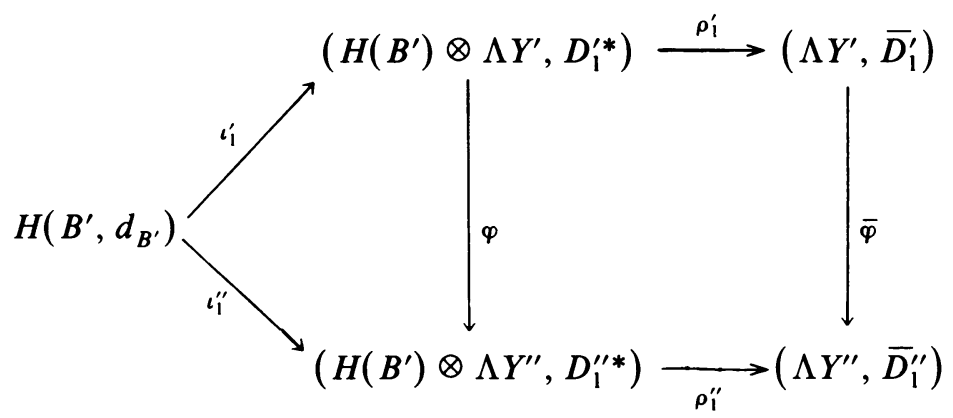

and from the relation $\zeta^{\prime \prime} \circ \varphi=\zeta^{\prime}$, we deduce that $\bar{\varphi}^{*}$ is an isomorphism, and from the minimality of the K. S. extensions [7] we deduce that $\bar{\varphi}$ and $\varphi$ are isomorphisms.

II.16. We state uniqueness of the E. M. model.

II.17. THEOREM. Let $\left(\mathscr{F}^{\prime}, \eta^{\prime}\right)$ and $\left(\mathscr{F}^{\prime \prime}, \eta^{\prime \prime}\right)$ be two E. M. models of a c.g.d.a. morphism $\gamma$ with same base $\left(\left(B^{\prime}, d_{B^{\prime}}\right), m\right)$. Then there exists an isomorphism $\left(\operatorname{Id}_{B^{\prime}}, \psi, \bar{\psi}\right)$ between $\mathscr{F}^{\prime}$ and $\mathscr{F}^{\prime \prime}$ such that $\psi\left(F_{p}^{\prime}\right)=F_{p}^{\prime \prime}, \bar{\psi}\left(\bar{F}_{p}^{\prime}\right)=\bar{F}_{p}^{\prime \prime}$ and $\eta^{\prime \prime} \circ \psi \sim \eta$ rel. $B^{\prime}$.

In view of Theorem II.14, we may assume the underlying formal models satisfy $G^{\prime}=G^{\prime \prime}$, and so that $\mathrm{II} .17$ reduces to

II.18. Proposition. Suppose that the E. M. models in II.17 have the same underlying formal model. Then there exists an isomorphism of $K$. S. extension $\left(\operatorname{Id}_{B^{\prime}}, \psi, \bar{\psi}\right)$ such that:

(i) $(\psi-$ Id $)\left(Y_{p}\right) \subset F_{p-1}(p \geqslant 0)$.

(ii) $\eta^{\prime \prime} \circ \psi \sim \eta\left(\right.$ rel. $\left.B^{\prime}\right)$,

where the total space of $\mathscr{F}^{\prime}\left(\right.$ resp. $\left.\mathscr{F}^{\prime \prime}\right)$ is $\left(B^{\prime} \otimes \Lambda Y, D^{\prime}\right)\left(\right.$ resp. $\left.\left(B^{\prime} \otimes \Lambda Y, D^{\prime \prime}\right)\right)$.

II.19. This last proposition can be proved as the second part of 4.4 in [8], with a few changes.

III. Geometric interpretation and examples.

III.1. If $\pi: E \rightarrow M$ is a continuous map, we define an E. M. model of $\pi$ to be any model of the c.g.d.a.'s homomorphism $A(\pi):\left(A(M), d_{M}\right) \rightarrow\left(A(E), d_{E}\right)$, when $A$ is the Sullivan function [13]. 
III.2. Recall [6 or 7] that a rational fibration

$$
F \stackrel{j}{\rightarrow} E \stackrel{\pi}{\rightarrow} M
$$

is a sequence of base point preserving continuous maps between pointed path connected topological spaces such that a certain condition on the minimal model is satisfied. Rational fibrations include [7] Serre fibrations of path connected spaces in which one of $H^{*}(M), H^{*}(F)$ is a graded finite type space and $\pi_{1}(M)$ acts nilpotently on each $H^{r}(F)$. We define an E. M. model of the rational fibration (*) to be any E. M. model of the c.d.g.a. homomorphism $A(\pi)$ : we have then a commutative diagram

$$
\begin{array}{ccccc}
\left(A(M), d_{H}\right) & \stackrel{A(\pi)}{\rightarrow} & \left(A(E), d_{E}\right) & \stackrel{A(j)}{\rightarrow} & \left(A(F), d_{F}\right) \\
m \uparrow & & \uparrow \eta & & \uparrow \bar{\eta} \\
\mathscr{F}:\left(B, d_{B}\right) & \stackrel{\iota}{\rightarrow} & (B \otimes \Lambda Y, D) & \stackrel{\rho}{\rightarrow} & (\Lambda Y, \bar{D})
\end{array}
$$

where $m^{*}, \eta^{*}$ and $\bar{\eta}^{*}$ are isomorphisms (the last condition is true since we suppose (*) to be rational). Thus the E. M. fibre $(\Lambda Y, \bar{D})$ is a K. S. model of $F$, which is in general neither minimal nor a filtered model of $F$ in the sense of [8] as will be seen in III.4.

III.3. For the underlying formal model $(\mathcal{S}, \zeta)$, we have the following commutative diagram

$$
\begin{array}{ccccc}
H^{*}(M) & \stackrel{\eta^{*}}{\rightarrow} & H^{*}(E) & \stackrel{j^{*}}{\rightarrow} & H^{*}(F) \\
m^{*} \uparrow & & \zeta \uparrow & & \uparrow \bar{\zeta} \\
H\left(B, d_{B}\right) & \stackrel{\iota_{1}}{\rightarrow} & \left(H(B) \otimes \Lambda Y, D_{1}^{*}\right) & \stackrel{\rho_{1}}{\rightarrow} & \left(\Lambda Y, \bar{D}_{1}\right)
\end{array}
$$

where $m^{*}$ and $\zeta^{*}$ are isomorphisms, but in general $\bar{\zeta}^{*}$ is not an isomorphism (see III.4).

$\left(\Lambda Y, \bar{D}_{1}\right)$ and its geometric realization are both called the formal fibre of $(*)$.

III.4. E. M. model of the Hopf fibration. Consider the Hopf fibration

$$
S^{1} \stackrel{j}{\rightarrow} S^{2 n+1} \stackrel{\pi}{\rightarrow} \mathrm{C} P^{n}
$$

The map $\pi$ induces between the minimal models the homomorphism

$$
f:\left(\Lambda\left(b, b^{\prime}\right), d_{B}\right) \rightarrow(\Lambda x, 0)
$$

defined by

$$
\begin{array}{rlrl}
|b| & =2, & & \left|b^{\prime}\right|=|x|=2 n+1, \\
f(b)=0, & & f\left(b^{\prime}\right)=x, \\
d_{B}(b) & =0, & & d_{B}\left(b^{\prime}\right)=b^{n+1} .
\end{array}
$$


So we can see, since $\bar{D}=\bar{D}_{1}$, that the E. M. spectral sequence of (0) collapses at the $E_{2}$ term, but is not totally degenerated since $\left[x \bar{b}+x^{\prime}\right] \neq 0 \in \bar{E}_{2}^{-1,6}$. On the right diagram we see, from [8], that the Eilenberg-Moore spectral sequence of the space $F$ does not collapse since $d_{2}: E_{2}^{-2,9} \rightarrow E_{2}^{0,8}$ is nonzero.

III.11. Applications to formality of fibre, base, and total space. Recall that a rational fibration (*) is called weakly homotopically trivial [14] (W.H.T.) if $\pi_{\psi}(E)=\pi_{\psi}(M)$ $\oplus \pi_{\psi}(F)$, that a space is formal [8] if its filtered model is isomorphic to the bigraded model and weakly formal [8] if the E. M. spectral sequence of the space collapses. So we have the following relations:

$$
\left\{\begin{array}{c}
M \text { and } F \text { are formal spaces } \\
(*) \text { is an H. T. fibration }
\end{array}\right\} \Leftrightarrow\left\{\begin{array}{c}
E \text { is a formal space } \\
(*) \text { is an H. T. fibration }
\end{array}\right\}
$$

$\left\{\begin{array}{c}M \text { and } F \text { are formal spaces } \\ (*) \text { is a C. T. and W.H.T. fibration }\end{array}\right\}$

$E$ is a weakly formal

(*) is an H. T. fibration

$E$ is a formal space (2)

$\left\{\begin{array}{c}E \text { is a formal space } \\ (*) \text { is a C. T. fibration }\end{array}\right\} \Rightarrow\left\{\begin{array}{c}(*) \text { is a W.H.T. fibration } \\ M \text { and } F \text { are weakly formal spaces }\end{array}\right\}$

$$
\left\{\begin{array}{c}
E \text { and } F \text { are formal spaces } \\
(*) \text { is a C. T. fibration }
\end{array}\right\} \Rightarrow(*) \text { is a H. T. fibration. }
$$

(1) and (3) follow from the lemma:

III.12. LEMMA. The following conditions are equivalent if (*) is a C.T. fibration:

(i) $E$ is weakly formal.

(ii) (*) is weakly homotopically trivial and $M$ and $F$ are weakly formal.

We prove this lemma in the following manner: Consider the Vigue model of (*) [15]

$$
\left(\Lambda Z, d_{B}\right) \stackrel{\iota}{\rightarrow}(\Lambda Z \otimes \Lambda X, D) \stackrel{\rho}{\rightarrow}(\Lambda X, \bar{D}) .
$$

It is an E. M. model and from III.8 its underlying formal model can be chosen so that $D_{1}^{*}=1 \otimes \bar{D}_{1}$ :

$$
H\left(\Lambda Z, d_{B}\right) \stackrel{\iota}{\rightarrow}\left(H\left(\Lambda Z, d_{B}\right) \otimes \Lambda X, 1 \otimes \bar{D}_{1}\right) \stackrel{\rho}{\rightarrow}\left(\Lambda X, \bar{D}_{1}\right)
$$

Whence $(\Lambda Z \otimes \Lambda X, D)$ is the filtered model of $E[8]$.

So, $\langle\langle E$ weakly formal $\rangle\rangle$ is equivalent to $\langle\langle(\Lambda Z \otimes \Lambda X, D)$ minimal $\rangle\rangle([8])$. This is equivalent to $\left\langle\left\langle(*)\right.\right.$ is a W.H.T. fibration and $\left(\Lambda Z, d_{B}\right)$ and $(\Lambda X, \bar{D})$ are minimal $\rangle>$.

III.13. Counterexample for (2). Consider the rational fibration

$$
S^{3} \vee S^{3} \rightarrow E \rightarrow S^{3} \times S^{5}
$$


defined by its E. M. model with $\left(\Lambda\left(b, b^{\prime}\right), 0\right)$ as described below in low dimensions $\left(H^{>12}(E)=0\right)$ :

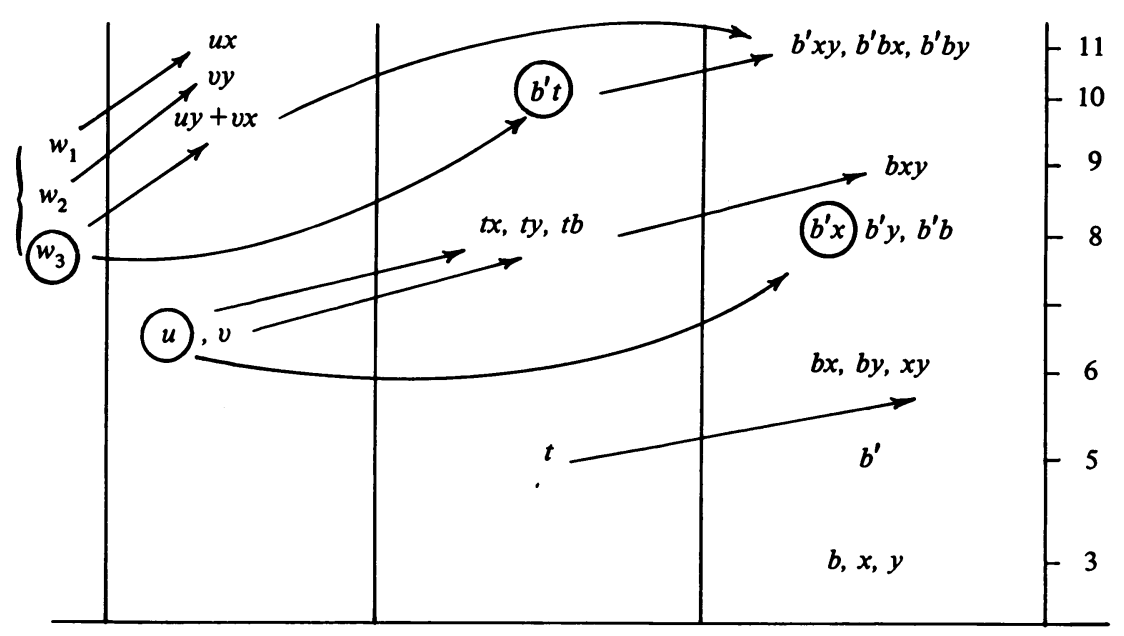

In this example, the base $M$ and the fibre $M$ are formal and coformal spaces, the fibration ( 0$)$ is W.H.T. (since $b$ and $b^{\prime}$ represent nontrivial elements of $\pi_{\Psi}^{*}(M)$ ). $E$ is not a formal space as it can be seen from the obstruction theory of [8], and therefore (0) cannot be homotopically trivial.

III.14. Proof of (4). Consider the E. M. model of (*) introduced in the proof of III.12. Since $E$ and $F$ are formal and (*) C. T., from III.7 and III.12,

$(\Lambda X, \bar{D})$ is a bigraded model of $F$,

$(\Lambda X \otimes \Lambda Z, D)$ is a bigraded model of $E$.

On the other hand, since (*) is C. T., there also exists

$$
\text { б: } H^{*}(F) \rightarrow H^{*}(E)
$$

such that $j^{*} \circ \sigma=\operatorname{Id}_{H^{*}(F)}$ (cf. [14]). So that:

(i) $\mathscr{F}$ is a K. S. minimal model of (*).

(ii) There exists a c.g.d.a. morphism $\tau$ such that the following diagram commutes:

$$
\begin{array}{ccc}
\left(H^{*}(F), 0\right) & \stackrel{\sigma}{\rightarrow} & \left(H^{*}(E), 0\right) \\
\uparrow & & \uparrow \\
(\Lambda X, \bar{D}) & \stackrel{\tau}{\rightarrow} & (\Lambda Z \otimes \Lambda X, D)
\end{array}
$$

(iii) $\rho^{*} \circ \tau^{*}=\mathrm{id}_{H(\Lambda X, \vec{D})}$.

Since $(\Lambda X, \bar{D})$ is minimal, $\alpha=\rho \circ \tau$ is an automorphism of $(\Lambda X, \bar{D})$. Then $\tau^{\prime}=\tau \circ \alpha^{-1}$ satisfies $\rho \circ \tau^{\prime}=\mathrm{id}_{\Lambda X}$ and using (i), from [14] we deduce that (*) is $\mathrm{H}$. T. 
Proof. Directly from the definitions (ii) is equivalent to (iii).

(a) In the formal model $(\mathcal{G}, \zeta)$ of $(*)$ consider

$$
\rho_{1}:\left(H(B) \otimes \Lambda Y, D_{1}^{*}\right) \rightarrow\left(\Lambda Y, \bar{D}_{1}\right) \text {. }
$$

If we suppose (ii), then $H_{+}\left(\Lambda Y, \bar{D}_{1}\right)=0$ and $H_{0}\left(\Lambda Y, \bar{D}_{1}\right)=H(\Lambda Y, D)$ so that $\rho_{1}^{*}=\rho_{2}=\left(\rho^{*}\right)$ is surjective. Therefore $j^{*}$ is surjective.

(b) Reciprocally, suppose (*) T.N.C.Z. From the Leray Hirsch theorem we deduce that $H(E)$ is a free $H(B)$-module. Hence, by II.4, $H(B) \otimes \Lambda Y_{0} / D_{1}^{*}\left(H(B) \otimes(\Lambda Y)_{1}\right)$ is also a free $H(B)$-module. More precisely, there exists a graded subspace $V$ of $Y_{0}$ such that the natural inclusion

$$
\varphi:(H(B) \otimes V, 0) \rightarrow(H(B) \otimes \Lambda Y, D)
$$

induces an isomorphism $\varphi^{*}$ between cohomologies. Filter both sides by the $H(B)$ degree we obtain two first quadrant spectral sequences. From the Moore comparison theorem (Sem. Cartan 54-55, p. 3-04) we deduce that

$$
\varphi_{\iota}^{0, *}: V \rightarrow H\left(\Lambda Y, \bar{D}_{1}\right)
$$

is an isomorphism of bidegree $(0,0)$, whence

$$
H_{+}\left(\Lambda Y, \bar{D}_{1}\right)=0 \text { and } H_{0}\left(\Lambda Y, \bar{D}_{1}\right)=H(\Lambda Y, D)(\cong V)
$$

so that $\left(\Lambda Y, \bar{D}_{1}\right)=0$ is the bigraded model of $F$.

III.8. Cohomologically or homotopically trivial fibrations. Recall [14] that a fibration (*) is cohomologically trivial (C. T.) if there exists an algebra isomorphism $f$ such that the following diagram commutes:

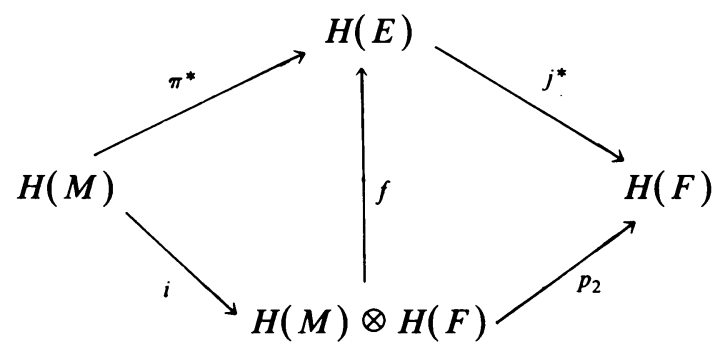

A fibration (*) is homotopically trivial (H. T.) if there exist a K. S. minimal model $(\mathcal{E}, \mu)$ and c.g.d.a. isomorphism $\varphi$ such that the following diagram commutes:

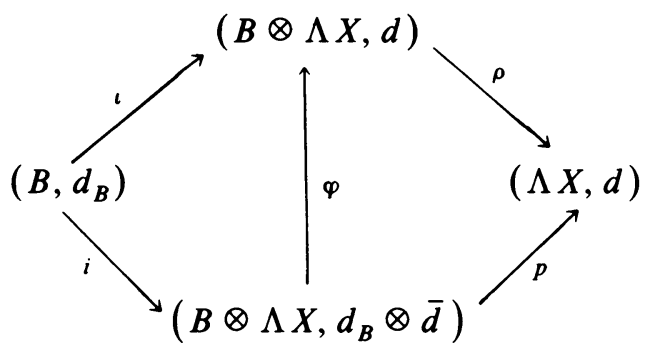

Evidently, we have the following result.

Proposition. The E. M. model (resp. the formal model) of an H. T. fibration (resp. C.T. fibration) can be chosen so that $D=d_{B} \otimes \bar{D}$ (resp. $\left.D_{1}^{*}= \pm 1 \otimes \bar{D}_{1}\right)$ and 
$(\Lambda Y, D)\left(\right.$ resp. $\left.\left(\Lambda Y, \bar{D}_{1}\right)\right)$ is the filtered model of $F$ (resp. the bigraded model of $H(F))$.

III.9. Eilenberg-Moore spectral sequences. Consider a c.g.d.a. homomorphism $\varphi$ : $\left(B, d_{B}\right) \rightarrow\left(B^{\prime}, d_{B^{\prime}}\right)$ and the push-out diagram,

$$
\begin{aligned}
& (B \otimes \Lambda Y, D) \quad \rightarrow \quad\left(B^{\prime} \otimes \Lambda Y, D^{\prime}\right)=\left(B^{\prime} \otimes{ }_{B}(B \otimes \Lambda Y), d_{B^{\prime}} \otimes D\right) \\
& \iota \uparrow \quad \uparrow \iota^{\prime} \\
& \left(B, d_{B}\right) \quad \stackrel{\varphi}{\rightarrow} \quad\left(B^{\prime}, d_{B^{\prime}}\right)
\end{aligned}
$$

then $\left(B^{\prime} \otimes \Lambda Y, D^{\prime}\right)$ is a filtered c.g.d.a. which gives a second quadrant spectral sequence $\bar{E}_{r}$ converging to $H\left(B^{\prime} \otimes \Lambda Y, D^{\prime}\right)$. With the definitions of [5], $(B \otimes \Lambda Y, D)$ is a Künneth object,

$$
D \operatorname{tor}_{B}\left(B^{\prime}, B \otimes \Lambda Y\right)=H\left(B^{\prime} \otimes \Lambda Y, D^{\prime}\right),
$$

and

$$
E_{r}\left(B^{\prime}, B, B \otimes \Lambda Y\right)=\bar{E}_{r}
$$

is the algebraic E. M. spectral sequence of the push-out diagram.

III.10. Example of an E. M. spectral sequence. Consider the rational fibration

$$
F \stackrel{j}{\rightarrow} \mathrm{C} P^{2} \stackrel{\pi}{\rightarrow} S^{4}
$$

where $\pi$ is the cellular application $C P^{1} \cup_{\varphi} D^{4} \rightarrow S^{4}$ which maps the 2-skeleton on the base point of $S^{4}$. The E. M. model of (0) and the filtered model of $F$ are described in low dimension by the two diagrams. For base one chooses $B=(1, b)$ with $b^{2}=0$.

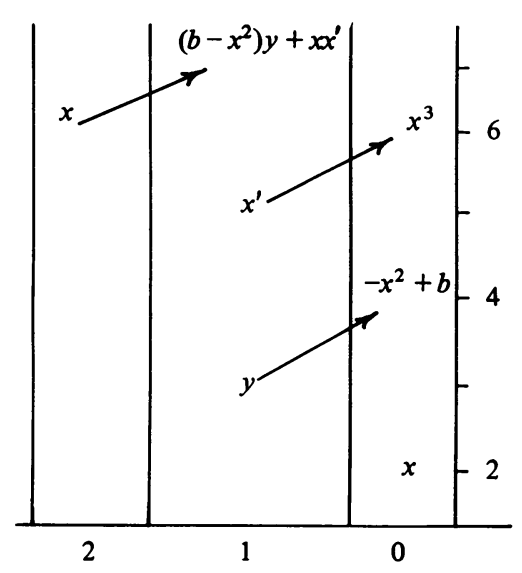

E. M. model of (1)

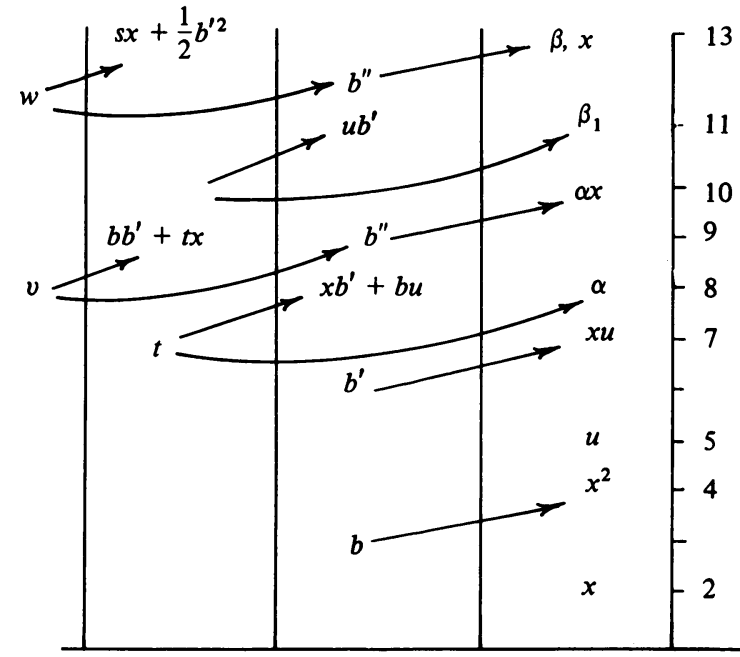

Filtered model of $F$ 
It follows at once from the definition that the E. M. model of $f$ is an E. M. model of $\pi$. The former has the form

$$
\mathscr{F}:\left(\Lambda\left(b, b^{\prime}\right), d_{B}\right) \stackrel{\iota}{\rightarrow}\left(\Lambda\left(b, b^{\prime}\right) \otimes \Lambda Y, D\right) \stackrel{\rho}{\rightarrow}(\Lambda Y, \bar{D})
$$

with

$$
\begin{array}{rlrl} 
& Y=Y_{0} \oplus Y_{1} \oplus Y_{2}, \\
Y_{0}=\mathbf{k} y_{0}, & Y_{1}=\mathbf{k} y_{1}, & Y_{2}=k y_{2}, \\
\left|y_{0}\right|=2 n+1, & \left|y_{1}\right|=1, & \left|y_{2}\right|=2 n, \\
D y_{0}=0, & D y_{1}=b, & D y_{2}=y_{0}-b^{\prime}+b^{n} y_{1} .
\end{array}
$$

We can verify that the E. M. fibre $(\Lambda Y, \bar{D})$ is not minimal since $\bar{D} y_{2}=y_{0}$ and not a filtered model in the sense of [8] since $Y_{1}$ and $Y_{2} \neq 0$. One can verify that this $\mathrm{E}$. M. model does not coincide with the Vigue model of $f$, although this base of $\mathscr{F}$ is also the filtered model of $C P^{n}$, since $b^{\prime}$ is here of filtration 0 and of filtration 1 in Vigué model.

III.5. The computation of the E. M. model becomes very easy when we use the Felix diagram [3] described below (in the particular case of the Hopf fibration):
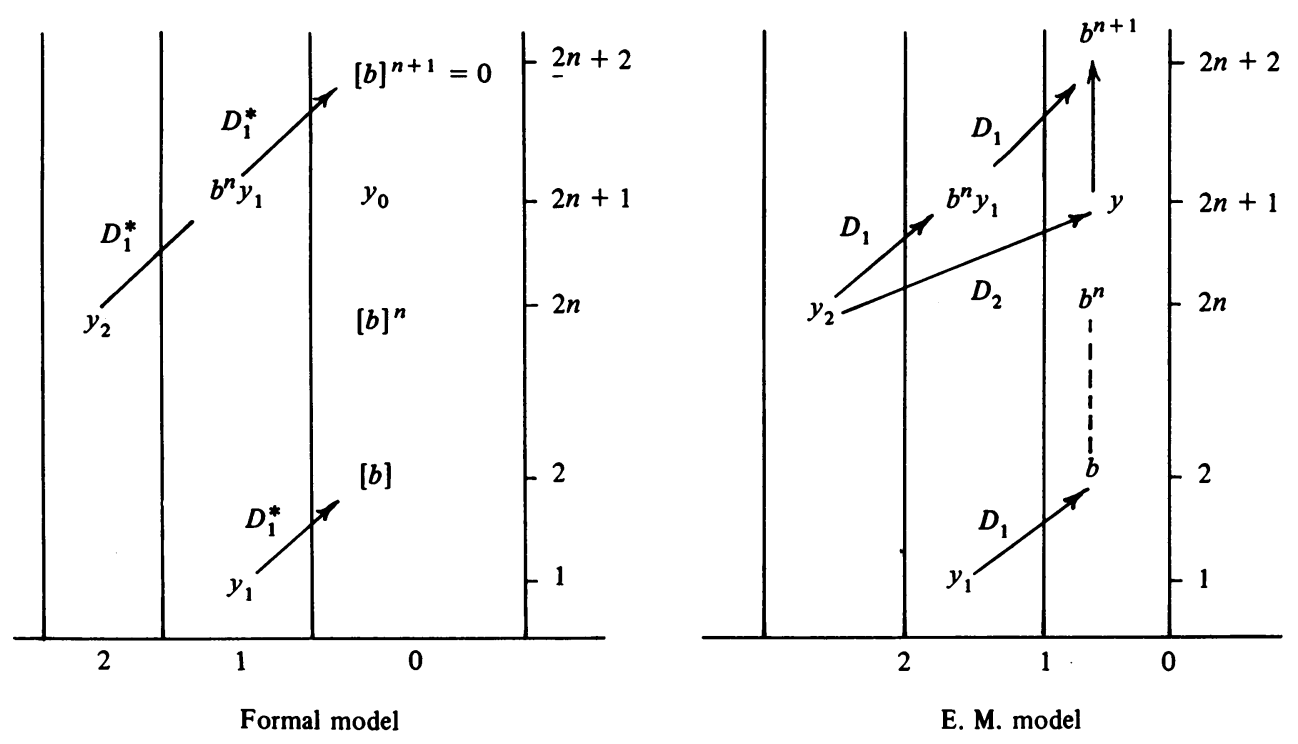

The left diagram is a Tate-Josefiak resolution of $H^{*}(E)$ as $H^{*}(M)$ module and the right diagram is a perturbation of the preceding when we replace $H^{*}(M)$ by a model of $M$. 
III.6. E. M. model of fibrations over a sphere. Consider the rational fibration, with base a sphere $S$

$$
F \stackrel{j}{\rightarrow} E \stackrel{\pi}{\rightarrow} S
$$

and $\nu:(\Lambda Z, \delta) \rightarrow\left(\Lambda(E), d_{E}\right)$ a filtered model of $E[8]$.

(a) Suppose that $S=S^{2 n+1}$ and let $((\Lambda b, 0), m)$ be a minimal model of $S^{2 n+1}$; then

(a-1): If $\pi^{*}([b])$ is decomposable in $H^{*}(E)$ (or zero), then

$$
\begin{gathered}
Y_{0}=Z_{0}, \quad Y_{1}=\mathbf{k} \bar{b} \oplus Z_{1}, \quad Y_{i}=Z_{i}, \quad i \geqslant 2, \\
D_{\mid \Lambda Z}=\delta, \quad D \bar{b}=b-\Phi_{0} \quad \text { and } \nu\left(\Phi_{0}\right)-A(\pi) \circ m(b)=d_{E} \Omega .
\end{gathered}
$$

(a-2): If $\pi^{*}[b]$ is indecomposable in $H^{*}(E)$, then

$$
Y_{0} \oplus \mathbf{k} \Phi_{0}=Z_{0}, \quad Y_{i}=Z_{i}, \quad i \geqslant 1,
$$

$D$ is defined by the isomorphism

$$
\varphi:(\Lambda Z, \delta) \rightarrow(\Lambda b \otimes \Lambda Y, D)
$$

with $\varphi \mid Y=$ id and $\varphi(b)=\Phi_{0}$.

(b) Suppose that $S=S^{2 n}$ and let $\left(\left(\Lambda\left(b, b^{\prime}\right), d\right), m\right)$ be a minimal model of $S^{2 n}$ with $d b^{\prime}=b^{2}$. Choose $\varphi:\left(\Lambda\left(b, b^{\prime}\right), d\right) \rightarrow(\Lambda Z, \delta)$ so that $\varphi(b)=\Phi \in \Lambda Z_{0}, \varphi\left(b^{\prime}\right)$ $=\Phi^{\prime} \in(\Lambda Z)_{<1}$ and $\nu \varphi \sim A(\pi) m$ rel. $\Lambda\left(b, b^{\prime}\right)$.

(b-1): If $\Phi^{\prime}$ is decomposable (then $\Phi$ is decomposable)

$$
\begin{gathered}
Y_{0}=Z_{0}, \quad Y_{1}=Z_{1} \oplus \mathbf{k} \bar{b}, \quad Y_{2}=Z_{2} \oplus \mathbf{k} \overline{b^{\prime}}, \quad Y_{i}=Z_{i}, \quad i \geqslant 3, \\
D_{\mid \Lambda Z}=\delta, \quad D \bar{b}=b-\Phi, \quad D \overline{b^{\prime}}=\left(\Phi^{\prime}-b^{\prime}\right)+(b+\Phi) \bar{b} .
\end{gathered}
$$

(b-2): If $\Phi^{\prime}$ is indecomposable and $\Phi$ is decomposable

$$
Y_{0}=Z_{0}, \quad Y_{1}=Z_{1} \oplus \mathbf{k} \bar{b}, \quad Z_{2}=Y_{2} \oplus \mathbf{k} \Phi^{\prime}, \quad Y_{i}=Z_{i}, \quad i \geqslant 3,
$$

$D \bar{b}=b-\Phi, \Psi:\left(\Lambda\left(b, b^{\prime}\right) \otimes \Lambda Y, D\right) \rightarrow(\Lambda Z, b)$ is c.g.d.a.'s isomorphism such that $\left.\Psi\right|_{Y_{1}}: Y_{1} \rightarrow Z_{1}$ and $\left.\Psi\right|_{Y_{i}}: Y_{i} \hookrightarrow Z_{i}$.

(b-3): If $\Phi^{\prime}$ is indecomposable and $\Phi$ is indecomposable

$$
\begin{gathered}
Z_{0}=Y_{0} \oplus \mathbf{k} \Phi, \quad Z_{1}=Y_{1} \oplus \mathbf{k} \Phi^{\prime}, \quad Y_{i}=Z_{i}, \quad i \geqslant 2, \\
D=\delta_{\mid \Lambda Y} .
\end{gathered}
$$

III.7. E. M. model of a T.N.C.Z. fibration. Recall that a fibration (*) is T.N.C.Z. if $j^{*}: H^{*}(E) \rightarrow H^{*}(F)$ is surjective. We give a shorter proof for the following Vigué result.

Proposition. If $H^{1}(M, \mathbf{k})=0$ and $H^{*}(M)$ or $H^{*}(F)$ finite type algebras then the following conditions are equivalent:

(i) The fibration (*) is T.N.C.Z.

(ii) The formal fibre of $(*)$ is the bigraded model of $F$.

(iii) The E. M. fibre of (*) is the filtered model of $F$. 
IV. Obstructions to k-realizability.

IV.1. Consider two c.d.g.a. morphisms

$$
\begin{aligned}
\alpha:\left(A, d_{A}\right) & \rightarrow\left(C, d_{C}\right), \\
\alpha^{\prime}:\left(A^{\prime}, d_{A^{\prime}}\right) & \rightarrow\left(C^{\prime}, d_{C^{\prime}}\right),
\end{aligned}
$$

and a quasi-isomorphism

$$
\gamma_{A}:\left(A, d_{A}\right) \rightarrow\left(A^{\prime}, d_{A}\right)
$$

IV.2. $\alpha$ and $\alpha^{\prime}$ are called $c$-equivalent if there exists a commutative diagram

$$
\begin{array}{ccc}
H\left(A, d_{A}\right) & \stackrel{\alpha^{*}}{\rightarrow} & H(C, d) \\
f_{A} \downarrow & & \downarrow f_{C} \\
H\left(A^{\prime}, d_{A^{\prime}}\right) & \underset{\alpha^{\prime *}}{\rightarrow} & H\left(C^{\prime}, d_{C^{\prime}}\right)
\end{array}
$$

where $f_{A}$ and $f_{C}$ are isomorphisms. The couple $\left(f_{A}, f_{C}\right)$ is called a c-equivalence.

IV.3. A $c$-equivalence $\left(\gamma_{A}^{*}, f_{C}\right)$ is called $\mathbf{k}$-realizable if there exists:

(i) A K. S. minimal model $(\mathcal{E}, \xi)$ of $\alpha$, with base $\left(\left(B, d_{B}\right), m_{B}\right)$.

(ii) A K. S. minimal model $\left(\mathcal{E}^{\prime}, \xi^{\prime}\right)$ of $\alpha^{\prime}$ with base $\left(\left(B, d_{B}\right), m_{B} \circ \gamma_{A}\right)$.

(iii) An isomorphism of K. S. extensions $\left(\operatorname{id}_{B}, \varphi, \bar{\varphi}\right)$ from $\mathcal{E}$ to $\mathcal{E}^{\prime}$ such that $\varphi^{*}=\left(\xi^{*}\right)^{-1} f_{C} \xi^{*}$.

IV.4. If $\left(\gamma_{A}^{*}, f_{C}\right)$ is $\mathbf{k - r e a l i z a b l e , ~ w e ~ h a v e ~ t h e ~ f o l l o w i n g ~ c o m m u t a t i v e ~ d i a g r a m : ~}$

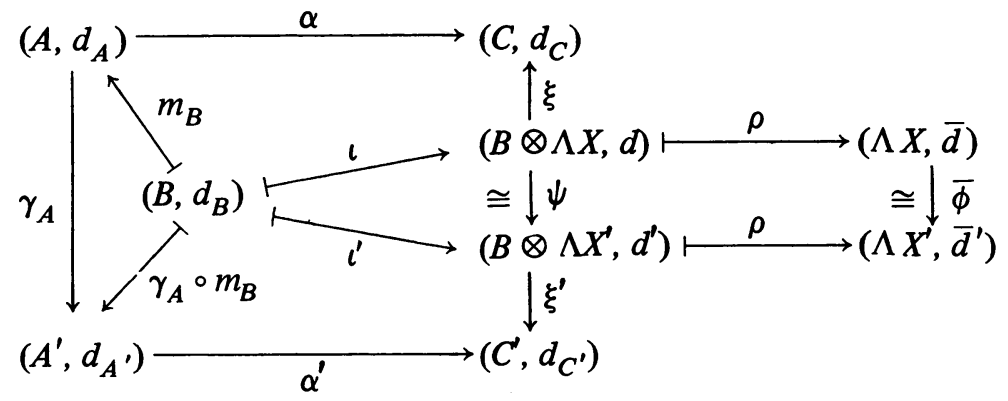

IV.5. We denote by $\beta$ a natural isomorphism (cf. [1])

$$
\beta: H \circ A \rightarrow H(\cdot, \mathbf{k}) \text {. }
$$

If $\alpha: S \mapsto T$ and $\alpha^{\prime}: S^{\prime} \rightarrow T^{\prime}$ are continuous maps, a couple $\left(f_{T}, f_{S}\right)$ is a $c$-equivalence between $\alpha$ and $\alpha^{\prime}$ if $\left(\beta_{T^{\prime}}^{-1} f_{T} \beta_{T}, \beta_{S^{\prime}}^{-1} f_{S} \beta_{S}\right)$ is a $c$-equivalence between $A(\alpha)$ and $A\left(\alpha^{\prime}\right)$.

A $c$-equivalence $\left(f_{T}, f_{S}\right)$ is k-realizable if $\left(\beta_{T^{\prime}}^{-1} f_{T} \beta_{T}, \beta_{S^{\prime}}^{-1} f_{S} \beta_{S}\right)$ is k-realizable.

IV.6. Proposition. (a) If there exists a commutative diagram

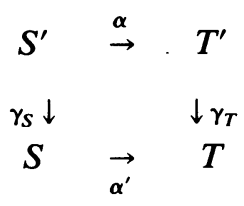


where $\gamma_{S}$ and $\gamma_{T}$ are rational homotopy equivalences then the c-equivalance $\left(\gamma_{T}^{*}, \gamma_{S}^{*}\right)$ is k-realizable.

(b) If $S, T, S^{\prime}, T^{\prime}$ are nilpotent spaces with rational cohomology of finite type, and if the c-equivalence $\left(\gamma_{T}^{*}, f_{S}\right)$ is $\mathbf{Q}$-realizable then there exists a commutative diagram

$$
\begin{array}{rrr}
S_{\mathbf{Q}}^{\prime} & \stackrel{\alpha_{\mathbf{Q}}}{\rightarrow} & T_{\mathbf{Q}}^{\prime} \\
\left(\gamma_{S}\right)_{\mathbf{Q}} \downarrow & & \downarrow \gamma_{T} \\
S_{\mathbf{Q}} & \underset{\alpha_{\mathbf{Q}}^{\prime}}{\rightarrow} & T_{\mathbf{Q}}
\end{array}
$$

where $\gamma_{T}$ is an homotopy equivalence and $S_{\mathbf{Q}}, T_{\mathbf{Q}}, S_{\mathbf{Q}}^{\prime}, T_{\mathbf{Q}}^{\prime}\left(\right.$ resp. $\left.\alpha_{\mathbf{Q}}, \alpha_{\mathbf{Q}}^{\prime},\left(\gamma_{S}\right)_{\mathbf{Q}}\right)$ are the rationalized spaces (resp. the rationalized maps).

IV.7. The proof of IV.6 is an immediate consequence of the unicity of K. S. minimal models in part (a) and of existence of a geometric realization functor in (b) [1].

IV.8. Suppose that $\left(\gamma_{A}^{*}, f_{C}\right)$ is a $c$-equivalence.

Fix notations by the following diagram, where: $(\mathscr{F}, \eta)$ is an E. M. model of $\alpha$ with base $\left(\left(B, d_{B}\right), m_{B}\right)$ and underlying formal model $(\mathcal{G}, \zeta)$.

$\left(\mathscr{F}^{\prime}, \eta^{\prime}\right)$ is an E. M. model of $\alpha^{\prime}$, with base $\left(B, d_{B}\right),\left(\gamma_{A} \circ m_{B}\right)$ and underlying formal model $\left(\mathcal{S}, f_{C} \circ \zeta\right)$. Obviously, from II.14 such a model $\left(\mathcal{F}^{\prime}, \eta^{\prime}\right)$ exists.

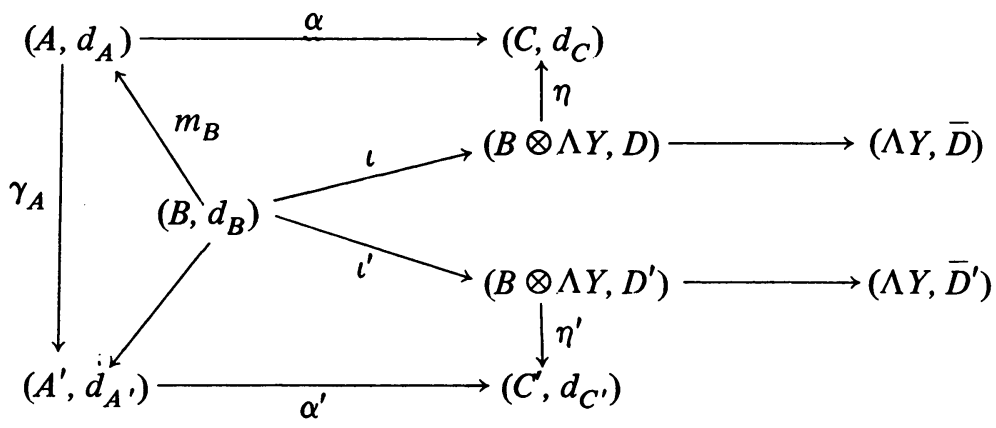

From II.18, we deduce

IV.9. Proposition. The c-equivalence $\left(\gamma_{A}^{*}, f_{C}\right)$ is $\mathbf{k}$-realizable if and only if there exists an isomorphism of $K$. S. extensions $\left(\operatorname{Id}_{B}, \Psi, \bar{\Psi}\right)$ such that:

(i) $(\Psi-$ Id $) Y_{p} \subset F_{p-1}, p \geqslant 0$.

(ii) $\left(\eta^{\prime}\right)^{*} \circ \Psi^{*}=f_{C} \circ \eta^{*}$.

IV.10. From the preceding theorem it is natural to define an $n$-realizer $\varphi$ as an isomorphism

$$
\varphi:\left(B \otimes \Lambda Y_{\leqslant n+1}, D\right) \rightarrow\left(B \otimes \Lambda Y_{\leqslant n+1}, D^{\prime}\right)
$$

such that $\varphi \circ \iota=\iota$ and $(\varphi-\operatorname{Id})\left(Y_{p}\right) \subset F_{p-1}, \forall p \in\{0,1, \ldots, n+1\}$.

If $\varphi$ is an $n$-realizer for $\left(\gamma_{A}^{*}, f_{C}\right)$, the degree +1 linear map

$$
O(\varphi): Y_{n+1} \rightarrow H\left(C^{\prime}, d_{C^{\prime}}\right)
$$


defined by $O(\varphi) \cdot y=\left[\eta_{1}^{\prime} \varphi D y\right]$ is called an obstruction element. We set $O_{n}\left(\gamma_{A}^{*}, f_{C}\right)$ $=\left\{O(\varphi) \mid \varphi\right.$ is an $n$-realizer of $\left.\left(\gamma_{A}^{*}, f_{C}\right)\right\}$ and denote by $\Delta_{n}$ the space of degree zero derivations $\theta$ of $B \otimes \Lambda Y_{<n}$ which satisfy:

(·) $D^{\prime} \theta=\theta D^{\prime}$

$(\cdot \cdot) \theta_{\mid B}=0$

$(\cdots) \theta\left(Y_{p}\right) \subset B \otimes\left(\Lambda Y_{<n}\right)_{<p-1}, \forall p \in\{0,1, \ldots, n\}$.

Consider then the linear map

$$
\gamma: \Delta_{n} \rightarrow \operatorname{Hom}^{1}\left(Y_{n+1}, H\left(C^{\prime}, d_{C^{\prime}}\right)\right)
$$

defined by $\gamma(\theta) \cdot y=\left[\eta^{\prime} \theta D^{\prime} y\right]$ and we denote by $\hat{O}_{n}\left(\gamma_{A}^{*}, f_{C}\right)$ the image of $O_{n}\left(\gamma_{A}^{*}, f_{C}\right)$ in the quotient space $\operatorname{Hom}^{1}\left(Y_{n+1}, H\left(C^{\prime}, d_{C^{\prime}}\right)\right) / \gamma\left(\Delta_{n}\right)$.

Following the proofs of [8], with the above notation and very few changes we obtain

IV.11. Proposition. Suppose that $\varphi$ is an $(n-1)$-realizer for $\left(\gamma_{A}^{*}, f_{C}\right)$ then $O_{n}\left(\gamma_{A}^{*}, f_{C}\right)=O(\varphi)+\gamma\left(\Delta_{n}\right)$.

IV.12. Proposition. An $(n-1)$-realizer $\varphi$ for $\left(\gamma_{A}^{*}, f_{C}\right)$ extends to an $n$-realizer iff $O(\varphi)=0$.

Corollary. If $\left(\gamma_{A}^{*}, f_{C}\right)$ is $(n-1)$-realizable, then $\left(\gamma_{A}^{*}, f_{C}\right)$ is n-realizable iff $O_{n}\left(\gamma_{A}^{*}, f_{C}\right)=\gamma\left(\Delta_{n}\right)$.

IV.13. THeOREM. (a) If $H^{*}\left(B, d_{B}\right)$ and $H^{*}\left(C, d_{C}\right)$ are of finite type, then $\left(\gamma_{A}^{*}, f_{C}\right)$ is realizable iff $\hat{O}_{n}\left(\gamma_{A}^{*}, f_{C}\right)=0, \forall n \in \mathbf{N}$.

(b) This obstruction theory is independent of the choice of the extension field $\mathbf{k}$ of $\mathbf{Q}$.

IV.14. Examples. (1) Let $T$ be the 19th-stage in a Postnikov tower of $\left(S_{a}^{5} \times S^{14}\right)$ $\vee S_{b}^{5}$ and $\alpha$ and $\alpha^{\prime}$ two continuous maps which represent respectively the two homotopy classes in $\pi_{17}(T)$ :

$$
\begin{gathered}
{\left[\left[\left[S_{a}^{5}, S_{b}^{5}\right], S_{b}^{5}\right] S_{b}^{5}\right],} \\
{\left[\left[\left[S_{a}^{5}, S_{b}^{5}\right], S_{b}^{5}\right] S_{a}^{5}\right]+\left[\left[\left[S_{a}^{5}, S_{b}^{5}\right], S_{a}^{5}\right] S_{b}^{5}\right] .}
\end{gathered}
$$

For any automorphism $f$ of $H\left(S^{17}, \mathbf{k}\right),\left(\mathrm{id}_{T}^{*}, f\right)$ is a $c$-equivalence which is not k-realizable since $\hat{O}_{3}(\mathrm{id}, f) \neq 0$.

(2) Let $\pi: E \rightarrow S^{3} \times S^{5}$, as in III.12, then $\pi$ is $c$-equivalent to the trivial fibration,

$$
\pi_{0}:\left(S^{3} \times S^{5}\right) \times\left(S^{3} \vee S^{3}\right) \rightarrow S^{3} \times S^{5}
$$

and from the Felix diagram described in III.13, we see that

$$
O_{1}\left(\left(\operatorname{id}_{S^{3} \times S^{3}}\right)^{*}, \operatorname{id}_{H\left(S^{3} \times S^{5}\right) \times H\left(S^{3} \vee S^{3}\right)}\right) \neq 0 .
$$

Since $\left(S^{3} \times S^{5}\right) \times\left(S^{3} \vee S^{3}\right)$ is a formal space, we deduce that any $c$-equivalence $\left(\left(\operatorname{id}_{s^{3} \times s^{5}}\right)^{*}, f\right)$ is not realizable. We find again that this fibration is not $h$-equivalent to the trivial fibration.

(3) Since $O_{n}\left(\gamma_{A}^{*}, f_{C}\right) \subset \operatorname{Hom}^{1}\left(Y_{n+1}, H\left(C^{\prime}, d_{C^{\prime}}\right)\right)$, clearly the conditions

(i) $Y_{p}^{n}=0$, if $n \leqslant l(p+1)$,

(ii) $H^{s}\left(C^{\prime}, d_{C^{\prime}}\right)=0$, if $s>N$, 
imply that there exists only a finite number of nonzero obstructions

$$
\left(\operatorname{Hom}^{1}\left(Y_{p+1}, H\left(C^{\prime}, d_{C^{\prime}}\right)\right)=0 \text {, if } p>(N-2) / l-2\right) \text {. }
$$

(4) (i) In particular, if (*) is a rational T.N.C.Z. fibration

$$
F \stackrel{j}{\rightarrow} E \stackrel{\pi}{\rightarrow} M
$$

and $(\mathscr{F}, \eta)$ an E. M. model of $\pi$, then $(\Lambda Y, \bar{D})$ is the filtered model for $F$ (III.7). Hence if $H^{r}(F)=0,1 \leqslant r \leqslant l$ and $H^{s}(E)=0, s>N=3 l+1$, then every obstruction is zero (cf. [8, Corollary 5.16]).

(ii) From (i) we deduce that if (*) if C. T. and if $H^{r}(E)=0,1 \leqslant r \leqslant l, r>3 l+1$, then $(*)$ is $H . T$.

IV.15. Remarks ON THE E. M. FIBRE. From IV.9, we deduce: If $\left(\gamma_{A}^{*}, f_{C}\right)$ is k-realizable then there exists an isomorphism

$$
\bar{\Psi}:(\Lambda Y, \bar{D}) \rightarrow\left(\Lambda Y, \bar{D}^{\prime}\right)
$$

such that

$$
(\bar{\Psi}-\mathrm{Id})\left(Y_{p}\right) \subset \bar{F}_{p-1}, \quad p \geqslant 0 .
$$

In particular, $(\Lambda Y, \bar{D})$ and $\left(\Lambda Y, \bar{D}^{\prime}\right)$ must have the same homotopy type.

For example, the Hopf map (III.4) and the constant map $S^{2 n+1} \rightarrow * \hookrightarrow \mathbf{C P} P^{n}$ are $c$-equivalent but any $c$-equivalence is not $\mathbf{k}$-realizable, since the geometric realization of E. M. fibre is $S_{\mathbf{Q}}^{1}$, and of the formal fibre is $K(\mathbf{Q}, 2 n) \times S_{\mathbf{Q}}^{1}$.

\section{Formalizable map.}

V.1. Given a c.g.d.a. $\left(A, d_{A}\right)$ and a minimal model $\left(\Re_{A}, m_{A}\right)$ of $\left(A, d_{A}\right)$, we call formalization of $\left(A, d_{A}\right)$ any homomorphism

$$
\theta_{A}: \mathfrak{N}_{A} \rightarrow H\left(A, d_{A}\right)
$$

such that

$$
\theta_{A}^{*}=m_{A}^{*}: H\left(\mathfrak{N}_{A}\right) \rightarrow H\left(A, d_{A}\right) .
$$

V.2. If $\left(A, d_{A}\right)$ is formal, the bigraded model in the sense of [8]

$$
\rho:(\Lambda Z, d) \rightarrow H\left(A, d_{A}\right)
$$

is a formalization of $\left(A, d_{A}\right)$.

V.3. Let $\alpha:\left(A, d_{A}\right) \rightarrow\left(A^{\prime}, d_{A^{\prime}}\right)$ be a homomorphism and $\theta_{A}$ be a formalization of $\left(A, d_{A}\right)$.

(i) $\alpha$ is $\theta_{A}$-formal if there exist a formalization $\theta_{C}$ and a homomorphism $\mathfrak{R}_{\alpha}$ such that the following diagram is homotopy commutative:

$$
\begin{array}{ccc}
\left(A, d_{A}\right) & \stackrel{\alpha}{\rightarrow} & \left(C, d_{C}\right) \\
m_{A} \uparrow & & \uparrow m_{C} \\
\Re_{A} & \stackrel{\Re_{a}}{\rightarrow} & \Re_{C} \\
\theta_{A} \downarrow & & \downarrow \theta_{C} \\
H\left(A, d_{A}\right) & \underset{\alpha^{*}}{H} & H\left(C, d_{C}\right)
\end{array}
$$


(ii) $\alpha$ is formalizable if there exists a formalization $\theta_{A}$ such that $\alpha$ is $\theta_{A}$-formal.

(iii) $\alpha$ is formal if for all formalizations $\theta_{A}, \alpha$ is $\theta_{A}$-formal.

V.4. A homomorphism

$$
\theta_{S}: \Re_{S} \rightarrow H(S, \mathbf{k})
$$

such that $\theta_{S}^{*}=\beta_{S} m_{S}^{*}$ (IV.5) is called a formalization of the topological space $S$.

A continuous map $\alpha: S \mapsto T$ is called $\theta_{T}$-formal (resp. formalizable, formal) if $A(\alpha):\left(A(T), d_{T}\right) \rightarrow\left(A(S), d_{S}\right)$ is $\theta_{A(T)}$-formal (resp. formalizable, formal).

V.5. REMARKS. (a) The definition V.3(ii) coincides with Definition 3.6 of [10]. In [16 and 4] formalizable maps are called formal maps.

(b) From definition, it is clear that if $\alpha$ is $\theta_{A}$-formal and if $\theta_{A} \sim \theta_{A}^{\prime}$, then $\alpha$ is $\theta_{A}^{\prime}$-formal. We prove in V.8(b) that $\theta_{A}$-formalization depends effectively on the homotopy class of $\theta_{A}$.

V.6. Proposition. The following conditions are equivalent:

(i) $\alpha$ is $\theta_{A}$-formal.

(ii) The c-equivalence $\left(m_{A}^{*}, m_{C}^{*}\right)$ between $\mathfrak{R}_{\alpha}$ and $\alpha^{*}$ is $\mathbf{k}$-realizable.

(iii) There exists an isomorphism (id, $\Psi, \bar{\Psi})$ between an E. M. model $(\mathscr{F}, \eta)$ of $\mathfrak{K}_{\alpha}$ with base $\left(\Re_{A}\right.$, id) and an E. M. model $\left(\mathcal{F}^{\prime}, \eta^{\prime}\right)$ of $\alpha^{*} \circ \theta_{A}$ with base $\left(\Re_{A}\right.$, id) such that $(\Psi-\mathrm{id})$ strictly lowers the filtration and $\left(\eta^{\prime}\right)^{*} \circ \Psi^{*}=m_{C}^{*} \circ \eta^{*}$.

V.7. The proof of V.6 results directly from the unicity of K.S. minimal models, and from IV.9.

The obstruction theory of IV.10, directly applied.

V.8. Particular case when $H\left(A, d_{A}\right)$ is a free c.g.a. Such c.d.g.a.'s $\left(A, d_{A}\right)$ are canonically formal; i.e. any two formalizations are homotopic, whence, if $\alpha$ is formalizable then $\alpha$ is formal.

In this case, we built a new obstruction theory.

Denote by $H\left(A, d_{A}\right)=\Lambda X,(\mathscr{F}, \eta):(\Lambda X, 0) \stackrel{\sim}{\rightarrow}(\Lambda X \otimes \Lambda Y, D) \stackrel{\rho}{\rightarrow}(\Lambda Y, \bar{D})$ an $\mathrm{E}$. M. model of $\Re_{\alpha}$.

Since $d_{B}=0, D=D_{1}+D_{2}+\cdots$ and $D_{1}^{*}=D_{1}$ (cf. II.2 and II.5), Proposition V.6 yields:

$\alpha$ is formal if and only if there exists an automorphism $\Psi$ of $\Lambda X \otimes \Lambda Y$ such that:

(i) $\Psi \circ \iota=\imath$,

(ii) $\Psi \circ D=D_{1} \circ \Psi$,

(iii) $\left(\Psi-\right.$ id) $Y_{p} \subset F_{p-1}, p=0,1, \ldots$

Let $\widehat{\operatorname{Der}}_{i}^{s}$ be the space of degree $s$ derivations on $\Lambda X \otimes \Lambda Y$ satisfying:

(a) $\theta \circ \iota=0$,

(b) $\theta\left(Y_{p}\right) \subset F_{p-i}, p=0,1, \ldots$,

$\hat{H}_{i}^{s}$ be the homology of the cochain complex

$$
\cdots \rightarrow{\widehat{\operatorname{Der}_{i-1}^{s-1}}}^{\rightarrow \operatorname{Der}_{i}^{s}} \stackrel{\hat{D}_{1}}{\rightarrow \widehat{\operatorname{Der}}_{i+1}^{s+1} \rightarrow \cdots}
$$

with $\hat{D}_{1}=\left[D_{1}, \cdot\right]$ and $[\cdot, \cdot]$ is the bracket of derivations. 
If we write $\Psi=$ id $+\Psi_{1}+\Psi_{2}+\cdots+\Psi_{p}+\cdots$ with $\Psi_{p}\left(Y_{k}\right) \subset(\Lambda Y)_{k-p}$ then $\Psi_{1} \in \operatorname{Der}_{1}^{0}$ and the condition $\Psi D=D_{1} \Psi$ implies

$$
D_{2}=D_{1} \Psi_{1}-\Psi_{1} D_{1}=\hat{D}_{1}\left(\Psi_{1}\right) \text {. }
$$

We put

$$
\hat{\omega}_{1}=\left[D_{2}\right] \in \hat{H}_{2}^{1}
$$

and the preceding condition implies that $\hat{\omega}_{1}=0$.

Reciprocally, suppose that $\hat{\omega}_{1}=0$, then there exists $\theta \in \widehat{\operatorname{Der}}_{1}^{0}$ such that $\left[D_{2}\right]=$ $\left[D_{1}, \theta\right]$. Then $e^{\theta}=\varphi$ is an automorphism of $\Lambda X \otimes \Lambda Y$ and $\varphi D \varphi^{-1}=D_{1}+D_{3}^{\prime}+D_{4}^{\prime}$ $+\cdots$.

This proves that $\varphi D=D_{1} \varphi$, up to the order two, and that $\hat{D}_{1}\left(D_{3}^{\prime}\right)=0$. Whence we define

$$
\hat{\omega}_{2}=\left[D_{3}^{\prime}\right] \in \hat{H}_{3}^{1}
$$

and the construction may be continued as in [3].

V.9. Remarks on E. M. FiBRe. (a) In the same way as in IV.15, if $\alpha$ is $\theta_{A}$-formal, there exists an isomorphism between the E. M. fibre and the formal fibre

$$
\bar{\Psi}:(\Lambda Y, \bar{D}) \rightarrow\left(\Lambda Y, \bar{D}_{1}\right)
$$

such that

$$
(\bar{\Psi}-\mathrm{id})\left(Y_{p}\right) \subset \bar{F}_{p-1}, \quad p \geqslant 0 .
$$

(b) From (a), we deduce the following sequence of strict implications:

( $\alpha$ is $\theta_{A}$-formal) $\Rightarrow$ (The E. M. fibre and the formal fibre have the same homotopy type) $\Rightarrow$ (The Eilenberg-Moore spectral sequence of $\alpha$ collapses at the $E_{2}$ term $\left.\left(d_{r}=0, r \geqslant 2\right)\right)$.

V.10. REMARK. In the same way as in V.8, we obtain obstructions $\tilde{\omega}_{i} \in \tilde{H}_{i}^{1}$ to the existence of the isomorphism $\bar{\Psi}$ defined in V.9(a).

Denote by: $\tilde{H}_{p}^{s}$ : the homology of the cochain complex

$$
\cdots \rightarrow \widetilde{\operatorname{Der}}_{p}^{s} \stackrel{\tilde{D}_{1}}{\rightarrow} \widetilde{\operatorname{Der}}_{p+1}^{s+1} \rightarrow \cdots
$$

$\widetilde{\operatorname{Der}}_{p}^{s}$ : the space of degree $s$ derivation on $\Lambda Y$ which lowers the filtration by $p$, and

$$
\tilde{D}_{1}=\left[D_{1},\right] \text {. }
$$

V.11. EXAmples. (1) The natural inclusion, $S^{3} \vee S^{2} \rightarrow S^{3} \times S^{3}$ is a formal map, as it can be seen from IV.14(3).

(2) Consider a continuous map $\alpha$ which represents the homotopy class $i_{5}+$ $\left\langle i_{2}, i_{2}, i_{2}\right\rangle \in \Pi_{5}\left(S^{5} \times \mathbf{C} P^{2}\right)$ where $i_{5}$ (resp. $i_{2}$ ) is the fundamental class of $S^{5}$ (resp. $\left.\mathbf{C} P^{2}\right)$ and $\left\langle i_{2}, i_{2}, i_{2}\right\rangle$ the Whitehead product. The formalization

$$
\theta_{T}:\left(\Lambda\left(b_{5}, b_{2}, b_{5}^{\prime}\right), d\right) \rightarrow \Lambda b_{5} \otimes b_{2} /\left(b_{2}^{3}\right) \cong H\left(S^{5} \otimes \mathbf{C} P^{2}, \mathbf{k}\right)
$$

is defined by

$$
\theta_{T}\left(b_{5}\right)=b_{5}, \quad \theta_{T}\left(b_{2}\right)=b_{2}, \quad \theta_{T}\left(b_{5}^{\prime}\right)=0 .
$$


Let $\gamma$ be an automorphism of $\left(\Lambda\left(b_{5}, b_{2}, b_{5}^{\prime}\right), d\right)$ such that

$$
\gamma\left(b_{5}\right)=b_{5}, \quad \gamma\left(b_{2}\right)=b_{2}, \quad \gamma\left(b_{5}^{\prime}\right)=b_{5}+b_{5}^{\prime} .
$$

Then, $\theta_{T}^{\prime}=\theta_{T} \circ \gamma \nsim \theta_{T}$ and $\theta_{T}^{*}=\theta_{T}^{\prime *}$. A direct computation proves that $\alpha$ is $\boldsymbol{\theta}_{T}^{\prime}$-formal, but not $\boldsymbol{\theta}_{T}$-formal.

(3) Let $\alpha: S \rightarrow S^{2 p+1}$ be a continuous map such that:

$S$ is a formal space [8],

$H\left(S^{2 p+1}, \mathbf{Q}\right)=\Lambda b$ and $\alpha^{*}(b)$ is indecomposable in $H^{*}(S, \mathbf{Q})$.

Then $\alpha$ is a formal map (V.8).

(4) Let $\alpha: S \rightarrow T$ be a continuous map such that:

$H(T)=\Lambda X$,

$S$ is a formal space,

$\alpha^{*}: H^{*}(T, \mathbf{Q}) \rightarrow H^{*}(S, \mathrm{Q})$ is injective.

Then $\alpha$ is a formal map (V.8).

(5) The natural inclusion $G_{n, k} \rightarrow G_{\infty, k}$ is a formal map.

(6) The map $\pi: \mathbf{C} P^{2} \rightarrow S^{4}$ defined in III.10 is formalizable and its homotopy fibre is not a formal space. This proves that formal fibre and E. M. fibre can be isomorphic without being formal algebras.

(7) Consider the continuous map $S^{2} \times S^{5} \rightarrow \mathbf{C} P^{2}$ defined by its minimal model

$$
\left(\Lambda\left(b_{2}, b_{5}\right), d_{B}\right) \stackrel{\iota}{\rightarrow}\left(\Lambda\left(b_{2}, b_{5}\right) \otimes \Lambda y_{3}, d\right) \stackrel{\rho}{\rightarrow}\left(\Lambda y_{3}, 0\right)
$$

with $d b_{5}=b_{2}^{3}$ and $d y_{3}=b_{2}^{2}$. The differential $D$ and $\bar{D}$ of the E. M. model are described in low degree below.
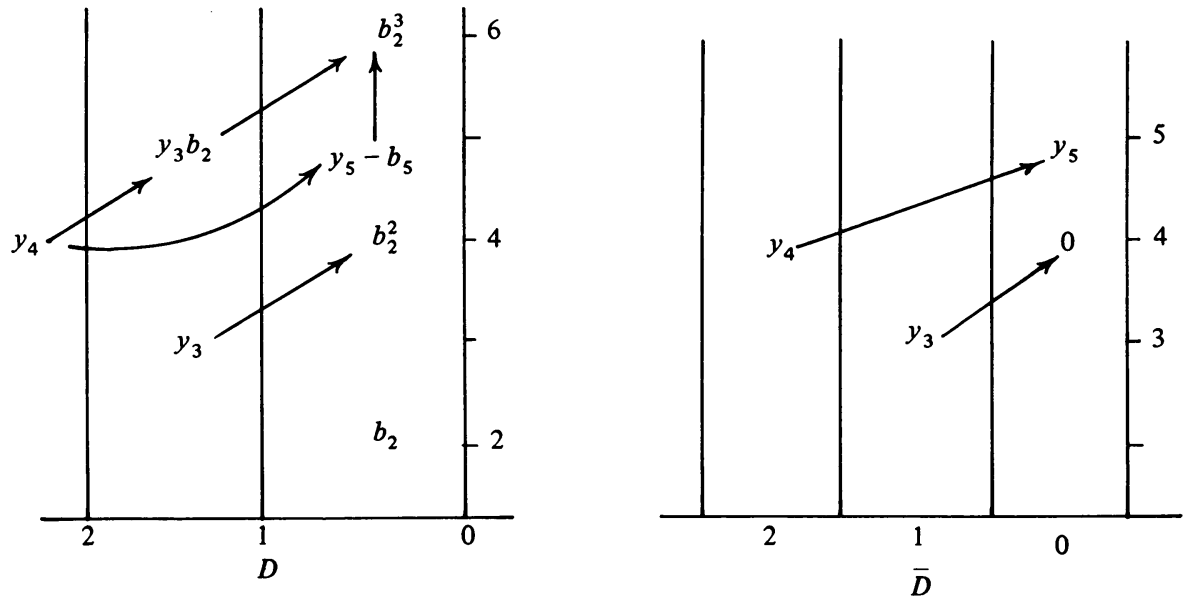

This map is not formalizable (V.9).

(8) The natural inclusions $i: S^{5} \rightarrow S^{5} \times \mathbf{C} P^{2}$ is formalizable but not formal (example 2) and the corresponding $\tilde{H}_{*}^{1}$ is zero.

(9) For $\pi: E \rightarrow S^{3} \times S^{5}$ as in III.13, all $\tilde{\omega}_{i}$ are zero although $\pi$ is not formalizable, and the E. M. spectral sequence of $\pi$ collapses. 


\section{REFERENCES}

1. A. K. Bousfield and W. K. A. M. Gugenheim, On the P. L. de Rham theory and rational homotopy type, Mem. Amer. Math. Soc. No. 179 (1976).

2. S. Eilenberg and J. C. Moore, Homology and fibrations, Comment Math. Helv. 40 (1966), 199-236.

3. Y. Felix, Dénombrement des types de K-homotopie-Théorie de la déformation, Nouveaux Mém. Soc. Math. France 3 (1980).

4. Y. Felix et D. Tanré, Sur la formalité des applications, Publ. IRMA Lille 3 (2) (1981).

5. W. K. A. M. Gugenheim and J. P. May, On the theory and applications of differential torsion products, Mem. Amer. Math. Soc. No. 142 (1974).

6. S. Halperin, Rational fibration, minimal models and fibring of homogeneous spaces, Trans. Amer. Math. Soc. 244 (1978), 199-223.

7. Lectures on minimal models, Preprint III, Univ. Sci. Tech. de Lille, 1977.

8. S. Halperin and J. Stasheff, Obstruction to homotopy equivalences, Adv. in Math. 32 (1979), 233-279.

9. J. M. Lemaire, Autopsie d'un meurtre, Ann. Sci. Ecole Norm. Sup. (4) 11 (1978), 93-100.

10. J. M. Lemaire et F. Sigrist, Sur les invariants d'homotopie rationnels liés à la L. S. catégorie, Comment Math. Helv. 56 (1981), 103-122.

11. J. C. Moore, Algébre homologique et homologie des espaces classifiants, Sém. Cartan E. N. S. 59/60 exposé 7.

12. J. D. Stasheff and M. Schlessinger, Deformation theory and rational homotopy type, (preprint).

13. D. Sullivan, Infinitesimal computations in topology, Publ. Inst. Hautes Etudes Sci. 47 (1977).

14. J. C. Thomas, Homotopie rationnelle des fibrés de Serre, Ann. Inst. Fourier (Grenoble) 31 (3) (1981); and C. R. Acad. Sci. Paris 290 (1980), 811-813.

15. M. Vigué, Réalisation de morphismes donnés en cohomologie et suite spectrale d'Eilenberg Moore, Trans. Amer. Math. Soc. 265 (1981), 447-484.

16. __ Formalité d'une application continue, C. R. Acad. Sci. Paris 289 (1979), 809-812.

U. E. R. de Mathematiques Pures et Appliquées, 59655 Villeneuve de'AscQ, France

Universite des SCiences et TeChNiques de Lille, 59655 Villeneuve de'AscQ, France 